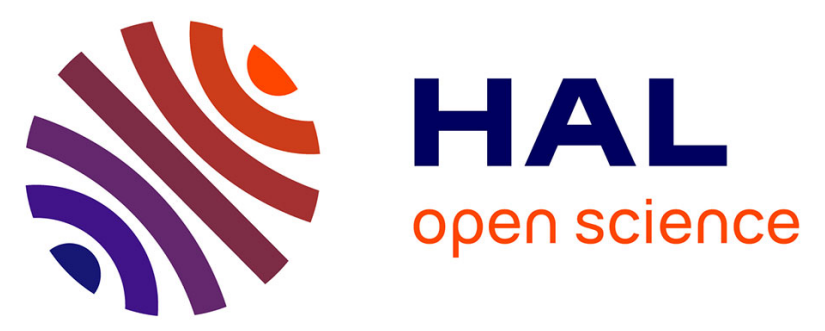

\title{
PIV and Electrodiffusion diagnostics of flow field, wall shear stress and mass transfer beneath three round submerged impinging jets
}

Kodjovi Sodjavi, Brice Montagné, Pierre Bragança, Amina Meslem, Paul Byrne, Cédric Degouet, Vaclav Sobolik

\section{To cite this version:}

Kodjovi Sodjavi, Brice Montagné, Pierre Bragança, Amina Meslem, Paul Byrne, et al.. PIV and Electrodiffusion diagnostics of flow field, wall shear stress and mass transfer beneath three round submerged impinging jets. Experimental Thermal and Fluid Science, 2016, 70, pp.417-436. 10.1016/j.expthermflusci.2015.10.004 . hal-01225592

HAL Id: hal-01225592

https://hal-univ-rennes1.archives-ouvertes.fr/hal-01225592

Submitted on 14 Jan 2016

HAL is a multi-disciplinary open access archive for the deposit and dissemination of scientific research documents, whether they are published or not. The documents may come from teaching and research institutions in France or abroad, or from public or private research centers.
L'archive ouverte pluridisciplinaire HAL, est destinée au dépôt et à la diffusion de documents scientifiques de niveau recherche, publiés ou non, émanant des établissements d'enseignement et de recherche français ou étrangers, des laboratoires publics ou privés. 


\title{
PIV and Electrodiffusion diagnostics of flow field, wall shear stress and mass transfer beneath three round submerged impinging jets
}

\author{
Kodjovi Sodjavi ${ }^{1}$, Brice Montagné ${ }^{1}$, Pierre Bragança ${ }^{1}$, Amina Meslem²*, Paul Byrne $^{2}$, \\ Cédric Degouet ${ }^{3}$, Vaclav Sobolik ${ }^{1}$
}

\begin{abstract}
${ }^{1}$ LaSIE, University of La Rochelle, Pôle Sciences et Technologie, avenue Michel Crépeau, 17042, La Rochelle, France

${ }^{2}$ LGCGM EA3913, Equipe Matériaux et Thermo-Rhéologie, Université Européenne de Bretagne, Université Rennes 1, IUT de Rennes, 3 rue du Clos Courtel, BP 90422, 35704 Rennes Cedex 7, France, amina.meslem@univ-rennes1.fr, *Corresponding author
\end{abstract}

${ }^{3}$ LaVision, Anna-Vandenhoeck-Ring 19, D-37081 Goettingen, Germany

Abstract - This paper reports on measurements of velocities, wall shear rates and mass transfer in an impinging round jet issued from a round nozzle. The effect of the nozzle shape on transfer phenomena was investigated. A round orifice perforated either on a flat plate $(\mathrm{RO} / \mathrm{P})$ or on a hemispherical surface $(\mathrm{RO} / \mathrm{H})$ was compared to a reference convergent nozzle (CONV). All the nozzles have the same exit diameter $D$. The exit volumetric flow rate was also conserved and led to the same Reynolds number based on the exit bulk velocity, $R e_{b}=5620$. The nozzle-to-wall distance was constant and equal to $2 D$.

The Particle Image Velocimetry technique (PIV) was used to capture the jet flow field. The limitations of the PIV technique in the vicinity of the target disc are addressed by using the electrodiffusion technique (ED) to obtain the wall shear rate distribution. The ED technique was extended for the measurement of local mass transfer distribution and global mass transfer on the target disc.

The whole velocity field, wall shear rates and mass transfer in the three impinging round jets were compared. It was shown that at constant volumetric flow rate, the use of an orifice nozzle not only improves wall shear rate, but also increases local and global mass transfer. The global mass transfer on a target disc of a $3.2 \mathrm{D}$ diameter is $25 \%$ and $31 \%$ higher for $\mathrm{RO} / \mathrm{H}$ and $\mathrm{RO} / \mathrm{P}$ nozzles, respectively, compared to the reference CONV nozzle.

The orifice nozzles generate narrower exit profiles relatively to the convergent nozzle. The vena contracta effect in orifice jets, more intense with $\mathrm{RO} / \mathrm{P}$ than with $\mathrm{RO} / \mathrm{H}$, generates an increase of the exit centerline 
velocity. The hemispherical surface of $\mathrm{RO} / \mathrm{H}$ nozzle stretches the flow at the exit and somewhat attenuates the vena contracta effect. The characteristic scale representation of the data confirms the origin of the observed differences between the three jets.

A link between the wall shear stress and the mass transfer is revealed. The wall shear rate and the mass transfer are in a close relationship with the near field flow features, themselves affected by the nozzle geometry. Time-resolved tomographic PIV technique reveals that the wall shear rate fluctuation is related to the dynamics of the jet coherent structures.

The instantaneous PIV fields indicates the formation of secondary vortices in the region where a secondary peak in local mass transfer emerges. The level of this secondary peak is sensitive to the nozzle shape. The higher is the jet acceleration, the more intense is the level of the secondary peak.

Keywords - Impinging round jet, time-resolved tomographic PIV, electrodiffusion, velocity field, wall shear rate, mass transfer

\section{List of symbols}

A hydrodynamic parameter $\mathrm{m}^{-1} \mathrm{~s}^{-1}$

C bulk concentration of active ions, $\mathrm{mol} / \mathrm{m}^{3}$

$D \quad$ nozzle jet exit nominal diameter

$D^{*} \quad$ characteristic jet exit diameter, $\mathrm{m}$

$D_{c} \quad$ diffusion coefficient of active ions, $\mathrm{m}^{2} / \mathrm{s}$

F $\quad$ Faraday constant, $96485 \mathrm{C} / \mathrm{mol}$

$f \# \quad$ lens aperture

$f \quad$ vortex shedding frequency, $\mathrm{Hz}$

$H \quad$ nozzle-to-plate axial distance, $\mathrm{m}$

I limiting diffusion current, A

$j \quad$ flux of active ions, $\mathrm{mol} / \mathrm{s}$

$k \quad$ coefficient of mass transfer, $k=I / S_{e l} n F c, \mathrm{~m} / \mathrm{s}$

$\mathrm{Nu} \quad$ Nusselt number

$n$ number of electrons involved in the electrochemical reaction

Q reconstruction quality

$\mathrm{Q}_{0} \quad$ Volumetric Flow rate, $\mathrm{m}^{3} / \mathrm{s}$

$R \quad$ electrode radius, $\mathrm{m}$

$r \quad$ radial distance measured from stagnation point, $\mathrm{m}$

Re Reynolds number based on the exit maximum velocity and characteristic diameter, $\operatorname{Re}=W_{0} D^{*} / v$

$R e_{b} \quad$ Reynolds number based on the exit bulk velocity and nominal diameter, $\operatorname{Re}_{b}=W_{b} D / v$

$R_{\theta} \quad$ Reynolds number based on the exit maximum velocity and the initial momentum thickness, $\operatorname{Re}_{\theta}=W_{0} \theta / v$

Sc $\quad$ Schmidt number, $S c=v / D$

$S_{e l} \quad$ active surface of the electrode, $\mathrm{m}^{2}$

Sh Sherwood number based on the nominal diameter, $S h=k D / D_{c}$

$S h^{*} \quad$ Sherwood number based on the characteristic diameter, $S h=k D^{*} / D_{c}$

$S t_{\theta} \quad$ Strouhal number, $S t_{\theta}=f \theta_{d} / W_{0}$ 
$S t_{e} \quad$ external excitation Strouhal number, $S t_{e}=f D / W_{0}$

$t$ time, $s$

$U \quad$ velocity in $x$ direction (normal velocity), $\mathrm{m} / \mathrm{s}$

$V \quad$ velocity in $y$ direction (spanwise velocity), $\mathrm{m} / \mathrm{s}$

$V_{r} \quad$ Radial velocity, $\mathrm{m} / \mathrm{s}$

$W \quad$ velocity in $z$ direction (streamwise velocity), $\mathrm{m} / \mathrm{s}$

$W_{0} \quad$ exit maximum velocity, $\mathrm{m} / \mathrm{s}$

$W_{b} \quad$ exit mean or bulk velocity, $\mathrm{m} / \mathrm{s}$

$(X, Y, Z)$ system of coordinates attached to the nozzle, $\mathrm{m}$

$\mathrm{Z}^{*} \quad$ axis normal to target wall with origin on the target wall, $\mathrm{Z}^{*}=\mathrm{H}-\mathrm{Z}(\mathrm{m})$

$\gamma \quad$ wall shear rate, $\mathrm{s}^{-1}$

$\gamma_{\text {MES }} \quad$ corrected wall shear rate, $\mathrm{s}^{-1}$

$\lambda_{2} \quad$ vortex detection criterion, $\mathrm{s}^{-2}$

$\mu \quad$ dynamic viscosity, $\mathrm{Pa} \mathrm{s}$

$\rho \quad$ density, $\mathrm{kg} / \mathrm{m}$

$\tau \quad$ wall shear stress, $\tau=\mu \gamma, P a$

$\tau^{\prime} \quad$ fluctuation of wall shear stress, $\mathrm{Pa}$

$v \quad$ kinematic viscosity, $\mathrm{m}^{2} / \mathrm{s}$

$\varepsilon \quad$ discharge coefficient

$\omega_{r} \quad$ radial vorticity component obtained by TPIV, $\mathrm{s}^{-1}$

$\omega_{\theta} \quad$ azimuthal vorticity component obtained by TPIV, $\mathrm{s}$

$\omega_{z} \quad$ axial vorticity component obtained by TPIV, $\mathrm{s}^{-1}$

$\omega_{Y} \quad$ azimuthal vorticity component obtained by $2 \mathrm{D}$ classical PIV, $\mathrm{s}^{-1}$

$\theta_{0} \quad$ initial shear layer momentum thickness

$\Delta \quad$ vector spacing

\section{Subscripts}

avg space average value

$S \quad$ stagnation point

$c \quad$ centerline value

\section{Introduction}

A fluid flow released against a surface can transfer large amounts of mass or thermal energy between the surface and the fluid. Enhanced heat and mass transfer for industrial devices rely essentially on impinging jet management. It provides an effective and flexible way for heat and mass transfer adjustment. A thorough research, beginning with contributions of Gardon et al. [1-3], concerned the heat and mass transfer in impinging jets, and an early observation has been made in [2] about the importance of jet turbulence in heat transfer processes. It was shown that some seemingly anomalous heat transfer phenomena can be explained as effects of the turbulence occurring in jets. Turbulence is generated by the jet itself and by external disturbances and varies significantly with the nozzle shape, the upstream conditions and the position within the jet. One decade after the observation of Gardon and Akrifat [2], Popiel and Boguslawski [4] claimed that 
nozzle exit configuration is the most important factor affecting the heat and mass transfer. Despite these first very significant indications, there are only a few studies dedicated to heat and mass transfer dependency on nozzle geometry. This section first reviews the literature regarding heat and mass transfers using impinging jets and presents the objectives of the study.

\subsection{Heat transfer in impinging jets}

Lee et al. [5] compared three round orifice nozzles with an exit jet Reynolds numbers in a range from 10000 to 30000 and nozzle-to-plate spacing $H$ in a range from $2 D$ to $10 D$, where $D$ is the exit nozzle diameter. The orifices were square-edged, standard-edged and sharp-edged. The square-edged orifice is straight hole with straight-through edges $\left(90^{\circ}\right.$ corners at the hole). The standard edged orifice has square edged corners at the entrance, and bevel edges at the outlet. The sharp-edged orifice is beveled through the entire thickness of the hole (with an angle of $45^{\circ}$ relative to the axis normal to the orifice plate). In the stagnation region, the sharp-edged orifice jet produces significantly higher heat transfer rates than either the standard-edged orifice jet or square-edged orifice jet. The effect of nozzle exit configuration on the stagnation point heat transfer is more sensible at shorter nozzle-to-plate spacing.

The literature reveals that for high exit Reynolds numbers and low nozzle-to-wall distances, two peaks are present on the radial distributions of local Nusselt number $N u$, produced by circular impinging jets. The first peak corresponds to the maximum of heat transfer rate and occurs approximately at the nozzle radius. In some investigations [5-8], the location of the first peak is observed from $r=0.5 \mathrm{D}$ to $r=0.7 \mathrm{D}$ for $H<4 \mathrm{D}$. This peak is attributed to the high turbulence intensity at the nozzle edge and to the direct impingement of large toroïdal Kelvin-Helmholtz $(\mathrm{K}-\mathrm{H})$ vortices originated in the mixing region.

The secondary peak occurs at the radial distance from the stagnation point ranging from $1.2 \mathrm{D}$ to $2.5 \mathrm{D}$ [5, 7-9]. The second peak is either attributed to the transition from laminar to turbulent boundary layer in the wall jet region [3] or to the unsteady separation of the induced secondary vortices that form near the wall under primary $\mathrm{K}-\mathrm{H}$ vortices [10]. Carlomagno and Andrea [11] give in their recent review of impinging jets a comprehensive description of secondary vortex dynamics. With increasing exit Reynolds number, the location of the secondary peak of $N u$ moves outwards from the stagnation point and the peak height increases [5].

\subsection{Wall shear stress and mass transfer in impinging jets}

Whereas numerous papers were published on the Nusselt number $N u$ distributions generated by impinging jets, only a few studies were dedicated to the analysis of the corresponding wall shear rate $\gamma$ distributions [6, 
12-14]. Comparison of available data of $N u$ and $\gamma$ reveals similarities in their distributions and in the number and the radial locations of their peaks. For high Reynolds number equal to 41600 and $H<4 D$, two peaks are evident in $\gamma$ (or in wall shear stress $\tau=\mu \cdot \gamma$ ) distribution [13]. Similarly to $N u$-distribution [5-8], the first peak in $\gamma$-distribution appears at the distance from the stagnation point ranging from $r=0.56$

$D$ to $r=0.74 D$, while the second peak is located at $r=1.9 D[13,15]$. The fact that peak locations in $\gamma$ distribution are closely matching with those of $\mathrm{Nu}$ - distribution suggests that the wall shear stress and the local heat transfer are closely linked.

If the round impinging jet is a well-documented flow for the kinematic and the heat transfer behaviors, the literature review reveals a lack of information on local mass transfer process in such a flow. The available few studies devoted to this subject have mainly used the electrochemical limiting diffusion current technique. Vallis et al. [16] used this method to study the radial distribution of mass transfer coefficient produced by a convergent impinging jet for exit Reynolds numbers ranging between 5000 and 30000 and for a nozzle to plate distance ranging from $5 \mathrm{D}$ to $20 \mathrm{D}$. The stagnation point mass transfer was found to be an increasing function of the exit Reynolds number and decreasing function of nozzle to plate distance. Based on the same technique, Kataoka et al. [12] performed measurements of local mass transfer for impinging convergent nozzle jet with an exit Reynolds number ranging from 4000 to 15000 and a nozzle-to-plate distance ranging from $2 \mathrm{D}$ to $10 \mathrm{D}$. The authors observed that the stagnation point mass transfer reaches a maximum when the nozzle-to-wall distance was equal to $6 D$ and noted that the mass transfer is enhanced owing to the velocity turbulence in the momentum boundary layer. Chin and Tsang [17] used the electrodiffusion method for the local mass transfer measurement from an impinging jet to the stagnation region on a circular disk electrode. The authors give the variation of the limiting current density as a function of the dimensionless electrode radius $R / D$ within a range from 0.02 to 3 . It was found that for $R / D$ from 0.1 to 1.0 for turbulent nozzle flow and from 0.1 to 0.5 for laminar nozzle flow, the electrode has a "uniform accessibility" to the diffusion ions. Beyond this uniform accessibility region the mass transfer rate was found to be a decreasing function of the lateral coordinate.

The connection of the heat or mass transfer phenomena with the large-scale structures which develop in the free jet region or with the subsequent flow dynamics in the stagnation and wall jet regions is now recognized $[7,10,18,19]$. Therefore, the control of large-scale structures in impinging jets is a key element in the strategy of heat and mass transfer optimization and control. The passive control based on nozzle geometry modifications is particularly attractive because of easy implementation in industrial applications.

Kristiawan et al. [20] have compared the performance of the cross-shaped orifice nozzle impingement jet with the reference convergent nozzle in terms of stagnation point mass transfer. They calculated mass 
transfer rate in the impingement region from the measured wall shear rate in the vicinity of the stagnation point under the assumption of uniform thickness of hydrodynamic and concentration boundary layer.

Meslem et al. [21] have compared a round plate orifice jet to a reference convergent nozzle jet in terms of wall shear rate and stagnation point mass transfer at a very low exit Reynolds number of 1360 . The same method as in [20] has been used in [21] for stagnation mass transfer calculation. It was concluded that the orifice jet enhances significantly the stagnation mass transfer compared to the reference convergent jet. The mass transfer distribution on the target and the global mass transfer were not measured in the previous study. Hence, the global performance of the orifice jet relatively to the convergent jet remains unproven.

\subsection{Objectives}

The present investigation is the continuation of a previous study [21]. It is considered herein a turbulent case with an exit Reynolds number of 5620 rather than the very low exit Reynolds number of 1360 considered in [23]. In our previous study, only the stagnation mass transfer has been provided and has been deduced from the slope of the radial wall shear rate distribution [20,21]. In the present study, the radial mass transfer distribution and the global mass transfer is measured directly. A round plate orifice, a reference convergent nozzle and an innovative geometry, i.e., a round orifice perforated on a hemispherical surface are considered to drive the jet flow. According to our literature review, the hemispherical nozzle has never been used for impinging jet generation. The hemispherical surface which is supporting the round orifice considered in the present study is intended to increase the stretching of the shear layer at the jet exit, supposing to create a more efficient jet dynamics for wall skin friction and mass transfer enhancement.

The study is conducted at constant exit area and constant volumetric flow rate for the three jets. This specific choice is related to the aimed Heating, Ventilation and Air Conditioning (HVAC) application, and specifically to the Personalized Ventilation aspect [22]. For other applications where the energy conservation is required, further investigations should be conducted at the same power input. The flow exit configuration and the downstream flow characteristics are investigated along with the resulting mass transfer distribution at the vicinity of the target plate. The nozzle-to-wall distance is kept constant at $H=2 D$ with $D=7.8 \mathrm{~mm}$ for each nozzle. This particular distance was selected considering that it corresponds approximately to the first half of the potential core length of a round free jet [23], where the K-H toroïdal vortices are well formed and are still well defined at the target placed at this distance.

The wall shear stress and the mass transfer on the target plate are measured using the electrodiffusion method (ED), the same method as we have used before in $[20,21]$ for wall shear stress measurements. The electrodiffusion method (ED) is based on the measurement of the limiting diffusion current (I) on a working 
electrode (probe) and is presented in detail in section 2.3. In the present study, this method is extended for local and global mass transfer measurements. Phares et al. [14] made a critical survey of different techniques used for the measurements of wall shear stress and concluded that the ED method provides the greatest accuracy of any indirect method. To our knowledge, Kataoka et al. [6] were the first to introduce this technique for the measurement of wall skin friction generated by an impinging jet. This method provides information on the wall shear rates whereas the velocity field is captured using Particle Image Velocimetry (PIV). Both techniques (ED and PIV) are complementary as the PIV fails at the vicinity of the wall due to the laser scattering by the solid surface.

Particle Image velocimetry (PIV) is used in this study to capture velocity fields in the free and the near wall jet regions of the impinging jet. Recall that velocity fields in impinging jet flow have been measured for the first time by Landreth and Adrian [24]. Two PIV techniques were used in the present study: (i) the classical 2D PIV providing high spatial resolution in a plane, allowing the investigation of the mean flow fields in the three jets; (ii) the time-resolved tomographic PIV applied in the reference case (CONV nozzle) allowing a 3D vision of the flow. Although the spatial resolution is lower compare to the 2D classical PIV, the dynamic of the coherent flow structures of the convergent jet is captured and related to the wall shear rate fluctuation.

The following is structured within two main Sections. Section 2 presents the employed experimental procedures and section 3 is dedicated to results analysis.

\section{Experimental procedures}

\subsection{Experimental setup and geometry of nozzles}

The experiments are conducted in a liquid-liquid jet impinging orthogonally onto a wall. A schematic diagram of its generation in a reservoir is shown in Figure 1 a. A gear pump (Ismatec with a GJ-N23 head) draws the liquid from a reservoir and delivers it to a nozzle. The liquid jet issued from the nozzle impinges a circular target disc provided with six electrodes (Figure $1 \mathrm{~b}$ ) which serve as the probes for electrodiffusion measurements. The temperature of liquid is controlled by a cooling coil within $\pm 0.2^{\circ} \mathrm{C}$.

The nozzle is screwed to a $200 \mathrm{~mm}$ length stainless steel tube with inner and outer diameters of 15 and 20 $\mathrm{mm}$, respectively. A honeycomb manufactured of a $7 \mathrm{~mm}$ thick disc by drilling 17 holes with a diameter of 2 $\mathrm{mm}$ was fitted in the tube inlet. The nozzle assembly was located in a support which allowed vertical movement for accurate alignment of the nozzle axis with the electrodes centre. The reservoir was placed on 
a sliding compound table (Proxxon KT 150) which allowed movement in the axial and transverse direction relative to the nozzle with a precision of $0.05 \mathrm{~mm}$.

(a)

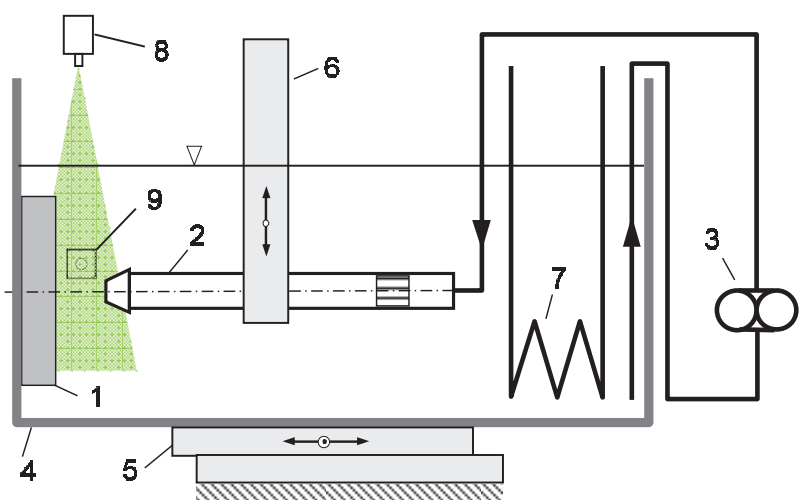

(b)

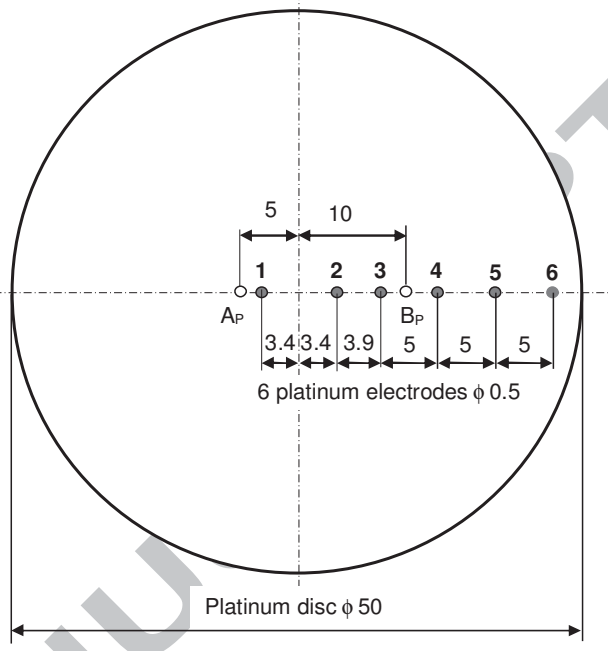

Figure $1 \mathrm{a}$ ): Diagram of apparatus: 1 target disc with electrodes, 2 tube with nozzle and honeycomb, 3 pump, 4 reservoir, 5 compound table, 6 nozzle holder, 7 cooling coil, 8 laser source with laser sheet in the mild plane of the nozzle, 9 FlowSense EO camera b): Target disc with electrodes row: 1-6 electrodes $\phi$ $0.5 \mathrm{~mm} . \mathrm{A}_{\mathrm{P}}$ and $\mathrm{B}_{\mathrm{P}}$ are the limits of stagnation point displacement.

The target was manufactured of a Plexiglas disc with a diameter of $100 \mathrm{~mm}$ and a thickness of $17 \mathrm{~mm}$ by first drilling holes to insert the electrodes. The platinum foil with a diameter of $50 \mathrm{~mm}$ and a thickness of $50 \mu \mathrm{m}$ (Figure $1 \mathrm{~b}$ ) was assembled centrally with the disc using Neoprene glue. Holes with a diameter $0.7 \mathrm{~mm}$ were drilled through the platinum foil as a continuation of the holes in the disc. The electrodes were manufactured of a $0.5 \mathrm{~mm}$ platinum wire which was coated electrophoretically using a deposit of a polymeric paint. After soldering the connection cables, the electrodes were glued with an epoxy resin into the disc, so that the tops of the platinum wires just projected above the platinum foil. The wires were then rubbed down flush with the surface of the platinum foil using progressively finer grades of emery paper. The last emery paper had a grit size of $10 \mu \mathrm{m}$. The whole surface was then polished using a fine dental paste. The resulting surface roughness was about $0.11 \mu \mathrm{m}$ which is much less than the Nernst diffusion layer thickness estimated at 10 $\mu \mathrm{m}$. Before each series of test, the electrode surfaces were polished and rinsed with distilled water. Nickel sheets introduced in the reservoir with an area of $0.15 \mathrm{~m}^{2}$ was used as the auxiliary electrode (anode). The area of the nickel sheets was 76 times superior to the platinum disc area. To measure only the phenomena which happen on the measuring electrode (cathode), the area of the auxiliary electrode (anode) should be 
large enough. Measured currents in our experiments did not change if the anode area was slightly varied which was the proof of the sufficiency of anode area.

The test fluid was an aqueous solution of $5 \mathrm{~mol} / \mathrm{m}^{3}$ potassium ferricyanide, $25 \mathrm{~mol} / \mathrm{m}^{3}$ potassium ferrocyanide and $1.5 \%$ mass potassium sulphate as supporting electrolyte. The solution had a density $\rho=$ $1006 \mathrm{~kg} / \mathrm{m}^{3}$, kinematic viscosity $\mathrm{v}=1.06 \cdot 10^{-6} \mathrm{~m}^{2} / \mathrm{s}$ and diffusivity $D_{C}=7.5 \cdot 10^{-10} \mathrm{~m}^{2} / \mathrm{s}$ at $20^{\circ} C$. The resulting Schmidt number was 1410 .

In this study, three round nozzles (Figure 2) having the same exit diameter $D=7.8 \mathrm{~mm}$ are compared: a convergent nozzle (CONV in Figure 2 a), a round orifice perforated on a flat plate (RO/P in Figure 2 b) and a round orifice perforated on a hemisphere $(\mathrm{RO} / \mathrm{H}$ in Figure $2 \mathrm{c}$ ). For $\mathrm{RO} / \mathrm{H}$ (Figure $2 \mathrm{c}$ ), the considered diameter $D$ corresponds to the projection on the plane of the curved orifice. The diameter corresponding to the curved free area of $\mathrm{RO} / \mathrm{H}$ is equal to $8.0 \mathrm{~mm}$. The flat and the curved orifices $(\mathrm{RO} / \mathrm{P}$ and $\mathrm{RO} / \mathrm{H}$, respectively) had the same thickness $e=0.5 \mathrm{~mm}$ (Figure $2 \mathrm{~b}$ and c). A convergent nozzle (Figure $2 \mathrm{a}$ ) had a conical shape with an area contraction $4: 1$ on a length of $17 \mathrm{~mm}$.

The exit Reynolds number based on the diameter $D$ and the jet bulk velocity $W_{b}\left(W_{b}=4 \mathrm{Q}_{0} / \pi D^{2}=0.77 \mathrm{~m} / \mathrm{s}\right)$ was $R e_{b}=5620$. The distance $H$ between the jet exit and the target wall was kept constant, $H=2 D$, for all the measurements. The coordinate system $(r, Y, Z)$ attached to the nozzle is shown in Figure 3. As sketched in this figure, the flow field may be divided into several regions. In the neighborhood of the stagnation point $\mathrm{S}$, the flow spreads in radial directions parallel to the wall. The development of the impinging jet flow field near the wall is typically divided in two regions: the stagnation region associated with the turning of the mean flow, $r / D<1$, and the radial wall jet region, $r / D>1$. 


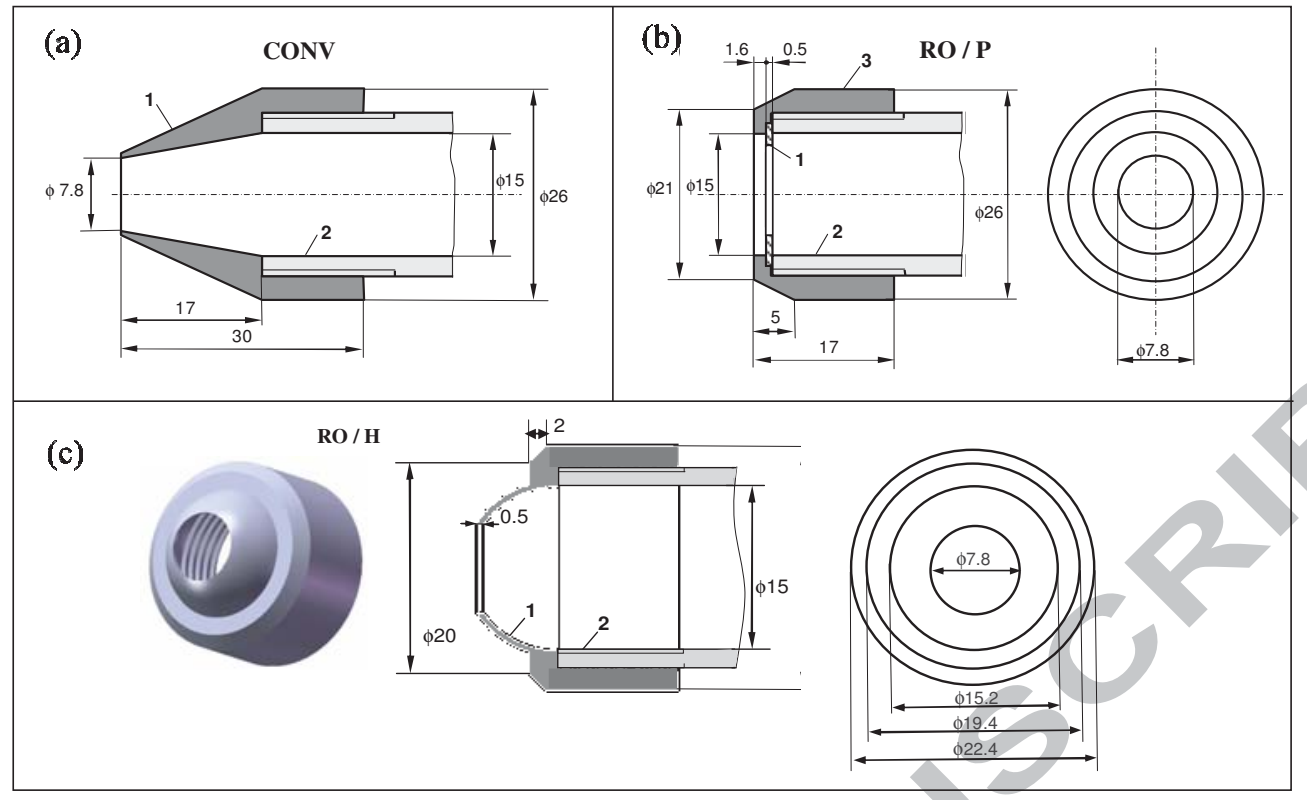

Figure 2: Sketch of nozzles: (a) 1 - Convergent nozzle (CONV), 2 - tube; (b) 1- Round Orifice on Plate (RO/P), 2 - tube, 3 - sleeve nut; (c) 1 - Round Orifice on Hemisphere (RO/H), 2 - tube

\subsection{PIV measurements}

\section{A) Classical 2D PIV Measurements}

Flow analysis has been carried out using PIV measurements. The PIV system, from the manufacturer Dantec, includes a Quantel BigSky 200 mJ double-pulsed Nd:Yag laser and a FlowSense EO (CCD) camera of $2048 \times 2048$ pixels resolution with pixel size of $7.4 \times 7.4 \mu \mathrm{m}^{2}$. The total field of view is about $2 D \times 6 D$ to cover free and wall jet regions (Figure 3) with an average resolution of 35.6 pixels $/ \mathrm{mm}$. The light sheet optics produces a laser sheet of less than $1 \mathrm{~mm}$ in thickness. The maximum acquisition frequency of the PIV system is $15 \mathrm{~Hz}$ which is lower than the shear layer frequency of considered jets. The seeding particles are glass hollow spheres of 9 to $13 \mu \mathrm{m}$ in diameter and $1.1 \mathrm{~g} / \mathrm{cm}^{3}$ in density. For each experiment, 500 couples of images are acquired. The recordings are analyzed through two different windows using DynamicStudio Dantec software. Firstly, the velocity distribution in the total field of view $(2 D \times 6 D)$ is calculated using an adaptive multi-grid correlation algorithm [25] handling the window distortion and sub-pixel window displacement $(128 \times 128,64 \times 64$, and $32 \times 32$ pixels $)$ and $50 \%$ overlapping. The resulted spatial resolution is $0.11 D \times 0.11 D$.

Secondly, to get a better resolution of velocity vectors in the radial wall jet region (Figure 3 ) the same algorithm is used with a final grid composed of $8 \times 64$ pixels interrogation windows and $50 \%$ overlapping. Hence, in the radial wall jet region the spatial resolution is $0.03 D \times 0.23 D$. The prediction-correction 
validation method of multi-grid algorithm identified on average less than $1 \%$ erroneous velocity vectors, which are replaced using a bilinear interpolation scheme. For all the experiments, the uncertainty of the measurement due to displacement error was estimated using the theoretical analysis of Westerweel [26]. When adding the global bias errors, the total uncertainty is estimated to be in the range of 2 to $3.5 \%$ outside the boundary layer. The uncertainty rises near the impinging plate due to laser scattering, so that the boundary layer is not accessible using PIV technique. This difficulty is bypassed using another measurement technique, which is the electrodiffusion (ED) method described in subsection 2.3.

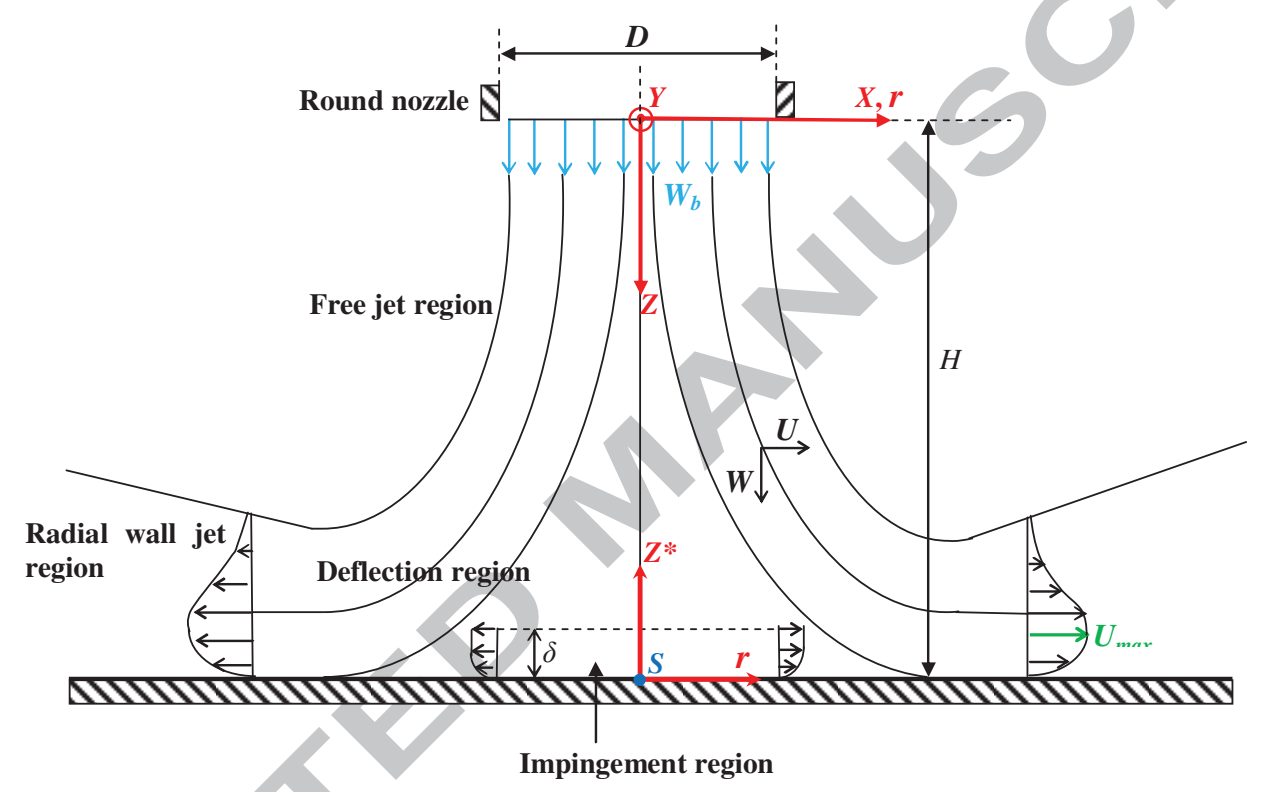

Figure 3: Schematic description of round impinging jet on a flat plate and associated system coordinates. The stagnation point is designated by $\mathrm{S}$.

B) Time-resolved tomographic PIV measurement

To get three-dimensional jet velocity fields and its dynamics, the time-resolved tomographic particle image velocimetry technique (TPIV) is used. This technique provides a spatial resolution fourteen times lower compared to the classical 2D PIV. However, its use allows the analysis of the possible link between the jet dynamics and the wall shear rate fluctuation. For brevity, the TPIV measurements are given for the convergent reference jet and the conclusions are extensible to the other considered two jets. 
The TPIV system consisted of high-repetition rate laser and three high-speed CMOS cameras (Phantom V9.1, 1600×1200 pixel2, 12 bit) equipped with Nikon objectives of $105 \mathrm{~mm}$ focal length. The sketch of the experimental setup is shown in Figure 4.

Two cameras were arranged in fowardscatter orientation with an angle approximately $20^{\circ}$ relative to the normal of the viewing face of the light volume. The third camera was oriented normal to the viewing face of the light volume. Scheimpflug adapters were used to align the mid-plane of the illuminated area with the focal plane and the lens apertures were fixed to $f^{\#}=16$. The test fluid was seeded with titandioxide filled polyamide12 fine powder particles of $20 \mu \mathrm{m}$ mean diameter. The illumination was provided by a pulsed, dual-cavity Nd:YLF laser with a power of $2 \times 10 \mathrm{~mJ}$. A beam expander (volume optics of LaVision), composed of two cylindrical lenses and a rectangular aperture, was used to generate a rectangular light volume. In order to increase the light, a mirror was inserted at the bottom of the reservoir to reflect the laser beam back to the measurement volume. The effective measurement volume was $2 D \times 6 D \times 5 D$ with an average resolution of 29.75 pixels $/ \mathrm{mm}$. The particle image density was approximately 0.04 particles/pixel. The imaging system was calibrated by the two-level spatial target (Type 7 target in the LaVision Software). In each of the two calibration planes, a third order polynomial fit was used to determine the transformation matrix which matched the viewing planes of the three cameras with an accuracy of approximately 0.18 pixels.

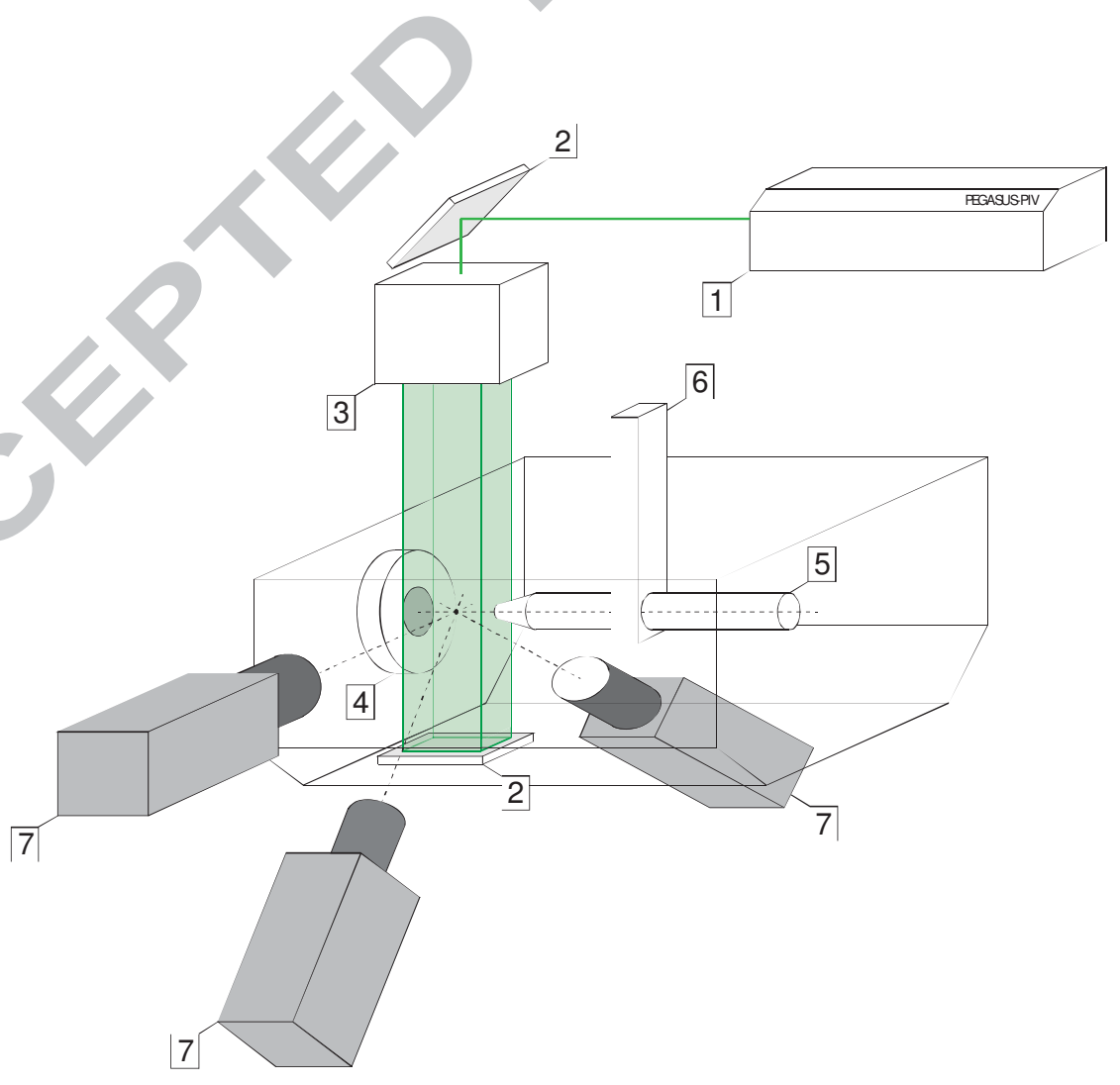


Figure 4: Schematic of the experimental setup of tomographic PIV: 1 laser head, 2 mirror, 3 beam expander with rectangular slit, 4 target disc, 5 tube with nozzle, 6 nozzle holder, 7 phantom V9.1 cameras, 8 laser beam

A number of 866 couples of images were recorded at a frequency of $1000 \mathrm{~Hz}$ with laser time interval $\delta t=250 \mu \mathrm{s}$.

Prior to the particle volume reconstruction, the original images were preprocessed to improve the reconstruction process. The main images preprocessing steps were described by Hain et al. [27]. A sliding minimum was subtracted from the images to reduce the background noise. A constant background due to laser reflecting or dirty Plexiglas was removed through an algorithmic mask, thus increasing the number of zero voxels. The particle image intensities were normalized by local image intensity, leading to similar particle intensity magnitude for the three cameras. Gaussian smoothing by $3 \times 3$ kernel was also applied. A geometric mask was used to define the boundaries of the calculated region.

The 3D particle positions within the volume were reconstructed using seven iterations of the Fast Multiplicative Algebraic Reconstruction Technique (Fast MART) algorithm provided by the LaVision software Davis 8.2.1. The volume was discretized with $520 \times 1544 \times 1188$ voxels and a pixel to voxel ratio of 1. The volume self-calibration [28] showed an initial particle based calibration error of up to 2-3 pixels. After self-calibration correction the errors are reduced to below 0.2 pixels. At the present particle image density and number of cameras, the reconstruction quality $Q$ is recognized to be above $0.75[29,30]$. In this condition, the reconstruction should be assumed to be sufficiently accurate [30].

The vector fields were obtained by performing multi-pass direct cross-correlation using a final interrogation volume of $64 \times 64 \times 64$ voxels $(0.28 D \times 0.28 D \times 0.28 D)$ and $75 \%$ overlap between adjacent interrogation boxes. The resulting vector spacing in each $3 \mathrm{D}$ velocity distribution is $0.07 D$. The spatio-temporal noisy fluctuations were reduced by using a second order polynomial filter over a kernel size of 5 grid nodes in space and 5 steps in time [7]. The spatial resolution of the filtered data remained equal to $0.28 \mathrm{D}$ whereas the temporal resolution was reduced to $4 \mathrm{~ms}$. The corresponding frequency of $250 \mathrm{~Hz}$ was approximatively five times greater than the vortex shedding frequency $(53 \mathrm{~Hz})$ of the studied jet flow.

The local mass conservation principle was used in numerous studies [7, 31, 32] to estimate the random velocity error in tomographic PIV measurements. In fact, when the flow is incompressible, the divergence of the $3 D$ velocity fields should be zero. However, this is not the case when there are measurement errors and numerical truncation in the spatial discretization. When a second order central difference scheme is used to calculate the velocity gradients, assuming uniform vector spacing $\Delta$, and uniform random error in all directions, the velocity gradient error is given by: 
$\delta\left(\frac{\partial u_{i}^{\prime}}{\partial x_{i}}\right)=\sqrt{\frac{3}{2 \Delta^{2}}} \delta(u)$

where $\delta\left(\frac{\partial u_{i}^{\prime}}{\partial x_{i}}\right)$ is the RMS fluctuation divergence and $\delta(u)$ the random error.

In this study the mean random error was found to be around 0.44 pixel, corresponding approximatively to $4.9 \%$ of the streamwise velocity in the free jet region. A random error varying between 0.60 and 0.42 pixel were reported by Atkinson et al. [31] and Buchner et al. [32] in turbulent boundary layer. The mean error of this measurement is in the same order of magnitude.

2.3 Electrodiffusion technique for wall shear rate and mass transfer measurements

\section{A) Wall shear rate}

The electrodiffusion method (ED) for wall shear rate measurement consists in using a working electrode flush-mounted on the wall to measure the limiting diffusion current. This technique was extensively described in Kristiawan et al. [20], El Hassan et al. [18] and Meslem et al. [21]. Thus, only a brief summary will be given here. The method is based on electrochemical redox reaction whose rate is very fast but the electric current is limited by the convective mass transfer on the measuring electrode (probe). For the total current through a circular electrode in a viscosimetric flow with a uniform wall-shear rate $\gamma_{M E S}$, the formula corresponding to the Leveque's equivalent equation for heat transfer was established by Reiss et al. [33] and is given by:

$$
I=\frac{0.884 \pi}{3^{1 / 3} \Gamma(4 / 3)} n F C \gamma_{M E S}^{1 / 3} D_{C}^{2 / 3} R^{5 / 3}
$$

where $C$ is the bulk concentration, $D_{C}$ the diffusion coefficient of active species, $F$ the Faraday constant, $n$ the number of electrons involved in the electrochemical reaction $\left(\mathrm{Fe}(\mathrm{CN})_{6}^{3-}+\mathrm{e}^{-} \Leftrightarrow \mathrm{Fe}(\mathrm{CN})_{6}^{4-}\right), R$ the radius of the electrode and $\Gamma$ the gamma function.

Eq. 2 was used to calculate the wall shear rate from the measured limiting diffusion currents for steady and quasi-steady flows. In practice the flows studied are often unsteady. Therefore, the wall shear rate measured using Eq. 2 do not take into account the unsteady nature of the diffusion boundary layer on the working electrode. This inertia effect acts as a filter and lead to an underestimation of the measured current fluctuations. A corresponding correction based on the dynamic theory of the electrodiffusion shear stress probe was proposed by Sobolik et al. [34]: 
$\gamma=\gamma_{M E S}\left[1+0.45 D_{C}^{-1 / 3} R^{2 / 3} \gamma_{M E S}^{-5 / 3} \frac{d \gamma_{M E S}}{d t}\right]$

where $\gamma_{M E S}$ is the wall shear rate calculated from measured currents using Eq. 2.

Let us recall that the basic assumptions for the derivation of the Leveque relationship (Eq.2) is that the probe is in a flow with parallel streamlines and uniform wall-shear rate $\gamma$. In the case of impinging jet, the streamlines in the wall vicinity spread radially from the stagnation point S (Figures 3 and 5) and the wallshear rate increases with $r$. Kristiawan et al. [20] have determined the drawbacks of application of this equation in the stagnation region for an electrode having a radius $R=0.25 \mathrm{~mm}$. At a radial distance $r=1$ $\mathrm{mm}$ from the stagnation point $\mathrm{S}$, the authors have found $2.1 \%$ error in wall shear rate. Taking into account the others parameters which can affect the wall shear rate, Meslem et al. [21] conclude that the error on the wall shear rate using Eq. 2 is less than $5 \%$ for $r \geq 1 \mathrm{~mm}$.

\section{B) Mass transfer}

As outlined before, the ED method is extended in the present study to local and global mass transfer measurements.

The limiting diffusion current is controlled by the transfer of active species to the working electrode. Under the assumption that the transfer resistance on the auxiliary electrode is negligible in comparison with that on the working electrode, the coefficient of mass transfer can be calculated using the following relation:

$k=\frac{j}{S_{e l} C}=\frac{I}{S_{e l} n F C}$

where $j=\iint_{S_{e l}}\left(-D_{C} \frac{\partial C}{\partial z^{*}}\right)_{z^{*}=0} d s$ is the flux of the active ions, $S_{e l}$ is the active surface of the working electrode.

The Sherwood number is then defined by:

$S h=\frac{k D}{D_{C}}$

Local and instantaneous wall shear rate and mass transfer were acquired separately. During the local wall shear rate measurements, the platinum wires worked as the cathode and the platinum disc (Figure $1 \mathrm{~b}$ ) with the nickel sheets as the anode. During the local and global mass transfer measurements the platinum wires and the platinum disc worked as the cathode and the nickel sheets as the anode. In this case, the platinum disc was maintained at the same potential as the worked electrodes; whereas the platinum disc was used to measure the global mass transfer, the platinum wires were used to measure the local mass transfer. 


\section{C) Measurements procedure}

Local and instantaneous wall shear rate and mass transfer were acquired separately using the electrodiffusion method (ED) described above in subsections A and B. The radial distributions of the wall shear rate and local mass transfer were obtained by moving the stagnation point $\mathrm{S}$ horizontally in the range limited by the points $A_{P}$ and $B_{P}$ on the target shown in Figure 1 b. Forty three displacements of the stagnation point with a step of $0.5 \mathrm{~mm}$ were performed to well capture the details of wall shear rate and local mass transfer profiles. Thereby, several values of the two quantities (wall shear rate and local mass transfer) measured by different electrodes at similar radial distances from the stagnation point were obtained.

The electrodes were calibrated before and after every series of measurements using a transient voltage step [35]. The result of this calibration, i.e. the Cottrell coefficient, which is proportional to $\left(C S_{e l} D^{1 / 2}\right)$, had a variation of about $\pm 4 \%$. The estimated errors were $\pm 8 \%$ and $\pm 4 \%$ for the wall shear rate and the Sherwood number respectively.

\section{Results and discussion}

\subsection{Mean flow characteristics}

Table 1 gives the initial conditions of the three studied flows. To take into account the vena contracta effect, which takes place in the orifice jet, additional parameters based on the characteristic jet diameter $D^{*}$ were introduced. The characteristic diameter is defined as follows [36]:

$$
D^{*}=D \sqrt{\varepsilon}
$$

where $\varepsilon=W_{b} / W_{0}$ is the discharge coefficient.

The obtained discharge coefficients are $0.94,0.72$ and 0.64 for the $\mathrm{CONV}, \mathrm{RO} / \mathrm{H}$ and $\mathrm{RO} / \mathrm{P}$ nozzle, respectively. A value of 0.61 is usually adopted for a sharp-edged orifice [36]. In the case of an impinging jet issued from multiple sharp-edged orifices Geers et al. [37] used a value of 0.71 .

To normalize the data presented in this section, two groups of parameters were considered. The first group includes the nominal diameter $D$, the jet bulk velocity $W_{b}$ and the corresponding Reynolds number $\operatorname{Re}_{b}$, and refers to "first normalization". The second group comprises the characteristic diameter $D^{*}$, the jet maximum velocity $W_{0}$ and the corresponding Reynolds number $R e$, and refers to "second normalization".

Figure 5 presents the mean velocity field of each round jet from classical 2D PIV measurements, superimposed to the corresponding contours of the azimuthal vorticity in the longitudinal plane. On the bottom of this figure, a zoom on the recirculation region is given for each jet in the window $0 \leq r / D \leq 1$ and $1.5 \leq Z / D \leq 2.0$. 
The entire fields (Figure $5 \mathrm{a}$, b1, and c1) clearly show that the free jet region is more contracted in RO/H and RO/P cases (Figure 5, b1, c1) than in CONV case (Figure 5 a1). From a detailed comparison of the orifice jets, it can be concluded that the RO/P nozzle generates greater contraction than RO/H nozzle. As shown in Figure 5 a2, b2 and c2, the contracted free jet region leads to a contracted deflection region.

For an axial position $Z$, the extent of the radial jet expansion in the free jet region is defined by the jet thickness $r_{0.1}$, which is the radial position in the jet where the axial velocity $W$ takes the value $0.1 W_{c} ; W_{c}$ is the centerline velocity at the same axial position $Z$. The streamwise evolution of the normalized jet thickness, $r_{0.1} / D$, is plotted in Figure 6 for each jet. As expected, the convergent jet is more expanded than the orifice jets (Figure 6 a). The comparison of the two orifice jets confirms that the RO/H nozzle attenuates the vena contracta effect, which characterizes orifice jets. When using the second normalization and considering the characteristic diameter $D^{*}$ as characteristic length (Figure $6 \mathrm{~b}$ ), the jet thickness of all three considered jets collapse well in the free jet region for $Z / D^{*} \leq 1.5$. In this normalization, the nozzle to plate distance $H$ takes different values as a function of the level of vena contracta effect; that is $H=2.1 D^{*}, 2.4 D^{*}$ and $2.5 D^{*}$ for the $\mathrm{CONV}, \mathrm{RO} / \mathrm{H}$ and RO/P nozzle jets, respectively. Near the wall, for $Z / D^{*}>1.5$, the jet thickness shows a different trend for the three jets due to different values of $H / D^{*}$; closer is the wall, faster is the jet deflection, leading to different jet thicknesses $r_{0.1} / D^{*}$. 
ACCEPTED MANUSCRIPT

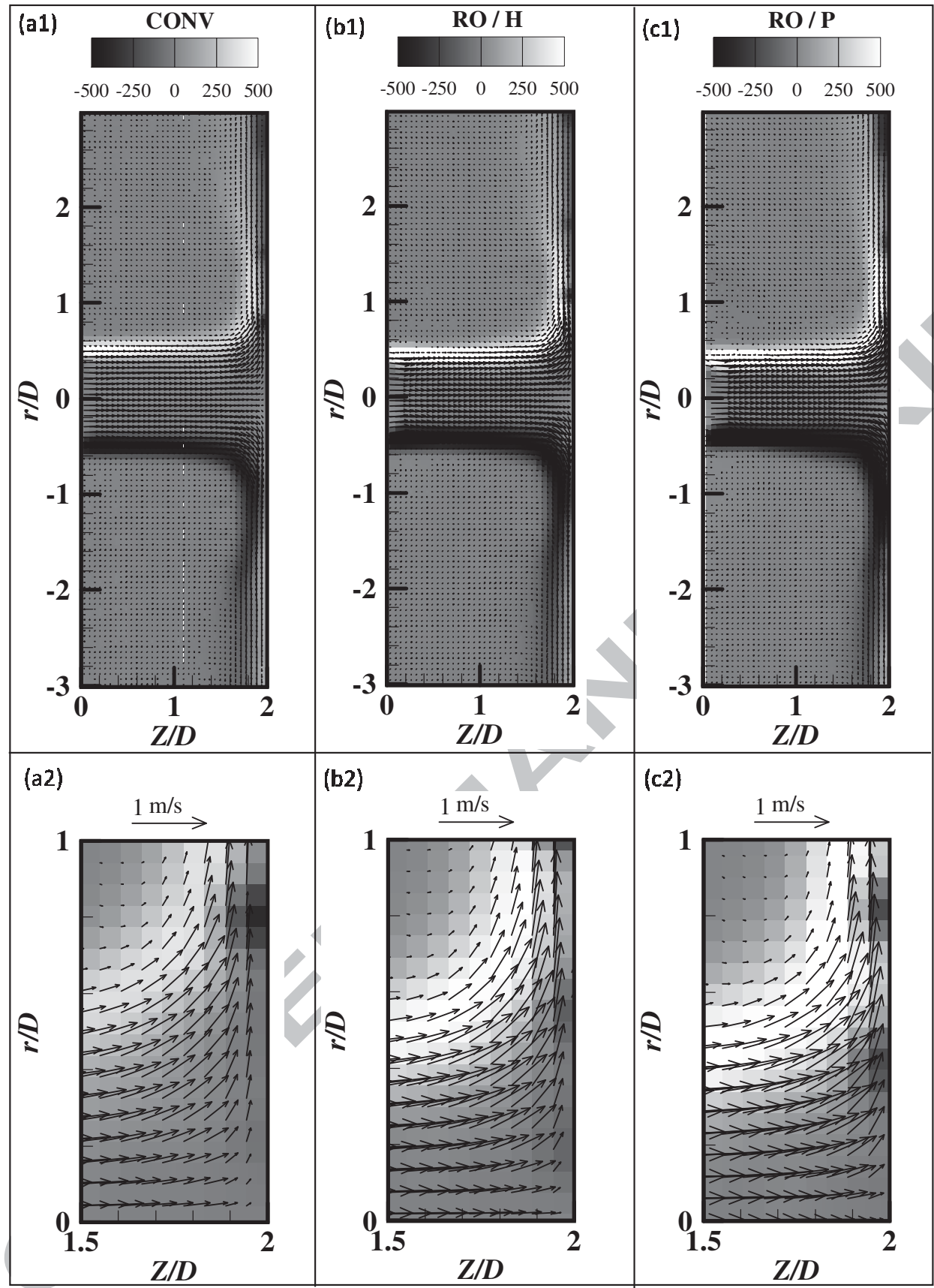

Figure 5: Mean vector field $\left(W \overrightarrow{e_{Z}}+U \overrightarrow{e_{r}}\right)$ and azimuthal vorticity $\omega_{Y}=\left(\frac{\partial U}{\partial Z}-\frac{\partial W}{\partial r}\right)$ contours (colormap) in longitudinal plane $Y=0$ of impinging jet - data from classical 2D PIV: (a) Convergent nozzle (CONV), (b) Round Orifice on Hemisphere (RO / H), (c) Round Orifice on Plane (RO / P); (1) entire field, (2) zoom on deflection region

Streamwise mean velocity changes along the jet centerline is given in Figure 7. As evidenced by Figure 7 a, the axial velocity achieves zero at the stagnation point $\mathrm{S}$. The value of jet bulk velocity $W_{b}$ reported in this figure highlights, when compared to the maximum centerline velocity $W_{0}$, the level of flow acceleration in each jet. The most accelerated flow is given by RO/P nozzle, followed by RO/H nozzle and then CONV 
nozzle (see also Table 1 where the values of $W_{0} / W_{b}$ are provided). When the maximum velocity $W_{0}$ and the corresponding characteristic diameter $D^{*}$ are used as normalized parameters for each jet (Figure $7 \mathrm{~b}$ ), the velocity decay $W / W_{0}$ is correlated to $H / D^{*}$ as was the case for the jet thickness $r_{0.1} / D^{*}$ (Figure $6 \mathrm{~b}$ ). However, if the origin of the jet axis is positioned on the target wall (Figure $7 \mathrm{c}$ ), the damping effect exerted by the wall is almost similar for the three jets, leading to nearly identical velocity changes.

Table 1: Initial conditions of the three studied flows

\begin{tabular}{l|c|c|c|c|c|c|c|c} 
Nozzle & $\begin{array}{c}W_{0} \\
(\mathrm{~m} / \mathrm{s})\end{array}$ & $\frac{W_{0}}{W_{b}}$ & $\frac{\left(U_{\text {max }}\right)^{\text {Peak }}}{W_{b}}$ & $\begin{array}{c}D^{*} \\
(\mathrm{~mm})\end{array}$ & $\operatorname{Re}=\frac{W_{0} D^{*}}{v}$ & $\begin{array}{c}\theta_{0} \\
(\mathrm{~mm})\end{array}$ & $\begin{array}{c}f \\
(\mathrm{~Hz})\end{array}$ & $\begin{array}{c}S t \theta= \\
f \theta_{0} / W_{0}\end{array}$ \\
\hline $\mathbf{C O N V}$ & 0.82 & 1.06 & 0.93 & 7.6 & 5850 & 0.192 & 53 & 0.012 \\
\hline $\mathbf{R O} / \mathbf{H}$ & 1.07 & 1.38 & 1.20 & 6.6 & 6680 & 0.168 & 66 & 0.010 \\
\hline $\mathbf{R O} / \mathbf{P}$ & 1.21 & 1.57 & 1.35 & 6.2 & 7100 & 0.163 & 76 & 0.010
\end{tabular}

The differences in jet contraction are visible also on the streamwise velocity profiles (Figure 8 a1 and b1), and on transverse velocity profiles as well (Figure $8 \mathrm{c1}$ ).

In the free jet region, at $Z=1.7 D$ (Figure $8 \mathrm{~b}$ ), axial velocity profile exhibits an "M shape" whereas at $Z=$ $0.5 D$ (Figure 8 a) the profile is flat. Hence, flow acceleration in the outer edge of the shear layer is a result of jet/wall interaction. Before impinging the wall, the jet accelerates in the outer edge (Figure 8 b1) and decelerates on the axis as visible on streamwise velocity changes along the jet centerline (Figure 7 a).

Considering the same positions $(Z=1.7 D$ and $Z=0.5 D)$, the second normalization is applied to the data of Figure 8 a1, b1 and c1, and the results are plotted in Figure 8 a2, b2 and c2. Near the jet exit at $Z=0.5 D$, the profiles collapse well. However, when approaching the wall at $Z=1.7 D$, a noticeable difference in magnitude of axial velocity (Figure $8 \mathrm{~b} 2$ ) and radial velocity (Figure $8 \mathrm{c} 2$ ) is visible between the three jets, due, as pointed before, to different nozzle-to-plate distances $H / D^{*}$

In Figure 8 a3, axial velocity profiles at $Z / D^{*}=0.5$ (i.e. at $Z=0.49 D, 0.42 D, 0.40 D$ for $\mathrm{CONV}, \mathrm{RO} / \mathrm{H}$ and $\mathrm{RO} / \mathrm{P}$, respectively) are plotted using the second normalization. As for $Z / D=0.5$ (Figure 8 a2), the profiles collapse well, meaning that apart the vena contracta effect, the jet exit velocity profiles are similar for the three jets.

To compare jets profiles before jet impingement at the same relative distance from the target wall, additional profiles were extracted at constant $Z^{*} / D^{*}$ equal to 0.3 , that is for $Z=1.71 D, 1.75 D, 1.76 D$ for $\mathrm{CONV}, \mathrm{RO} / \mathrm{H}$ and $\mathrm{RO} / \mathrm{P}$, respectively (Figure $8 \mathrm{~b} 3$ and $\mathrm{c} 3$ ). In this case, almost no difference appeared between the three 
jets, meaning that the jet scales $W_{0}$ and $D^{*}$ taking into account jet acceleration and jet contraction introduced by the round nozzle shape, are sufficient to model flow modification. This should remain valid as long as $H / D^{*}$ values are close, which is the case in the present study $\left(H / D^{*}\right.$ vary from 2.1 to 2.5$)$.

As outlined earlier, time-resolved tomographic PIV (TPIV) measurements were performed in the convergent reference jet to capture the jet flow dynamics. The validation of the TPIV measurements consists in their comparison in Figures 7 a and 8 a1, b1, c1 to those obtained with the 2D PIV. While rather good agreement between axial mean velocity profiles was observed in the jet core region (Figures $7 \mathrm{a}$ and $8 \mathrm{a} 1$ ), discrepancies appeared in the shear layer and flow deflection regions. At axial position $Z=1.7 D$, where the flow defects radially and creates a strong gradient region owing to the presence of the target wall, this discrepancy is about $18 \%$. This could be attributed to the difference of the spatial resolution between the two measurement techniques. Indeed, the 2D PIV measurements were obtained with a spatial resolution of $0.11 D \times 0.11 D \times 0.13 D$ (the third direction corresponds to the $1 \mathrm{~mm}$ thickness of the laser) while the spatial resolution of the $3 \mathrm{D}$ PIV was $0.28 D \times 0.28 D \times 0.28 D$, which is fourteen times greater than that in $2 \mathrm{D}$ PIV measurements. Hence, in the regions of strong gradients, the 3D PIV leads to an underestimation of velocities due to their spatial integration. Anyway, comparisons are satisfactory, which gives confidence in the tomographic PIV used in the present study.

Figure 9 gives the changes of maximum velocity $U_{\max }$ along the wall. The peak level of $U_{\max }$ is also linked to the jet acceleration level. However, the curves have similar shapes and the position of the peak of $U_{\max }, r=$ $0.95 \mathrm{D}$, seems to be insensitive to the contraction level of the jet flow (Figure 9a). When $U_{\max }$ is normalized by $W_{0}$ (Figure $9 \mathrm{~b}$ ), radial distributions of the maximum velocity in the wall jet region collapse into a single curve. The comparison of the obtained dimensionless distributions to those of round jets from the literature $[15,38,39]$ at a same normalized nozzle-to-plate distance, $H / D=2$, suggests a possible effect of jet exit Reynolds number on the level of $U_{\max } / W_{0}$. However, the radial position of the peak value remains almost unchanged with different values of Reynolds number and different round nozzle geometries. In fact, the round nozzle in $[15,39]$ is a long pipe and a convergent nozzle connected to a short pipe in [38]. 

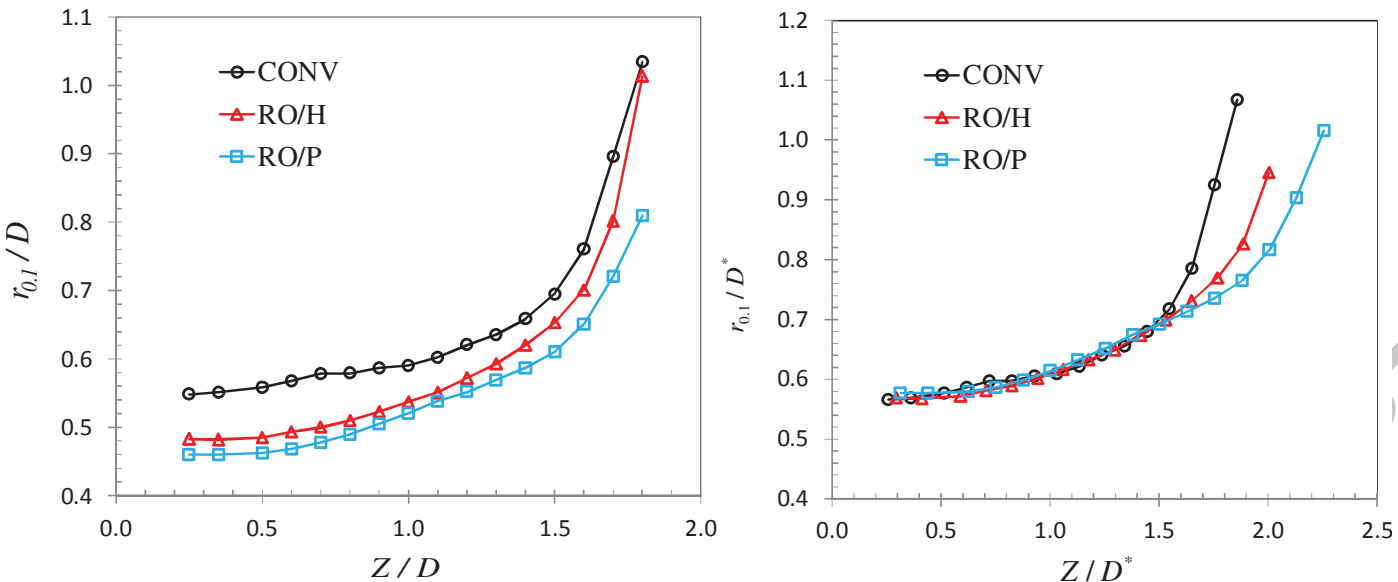

Figure 6: Growth of the jet thickness; (a) using the first normalization; (b) using the second normalization

(a)

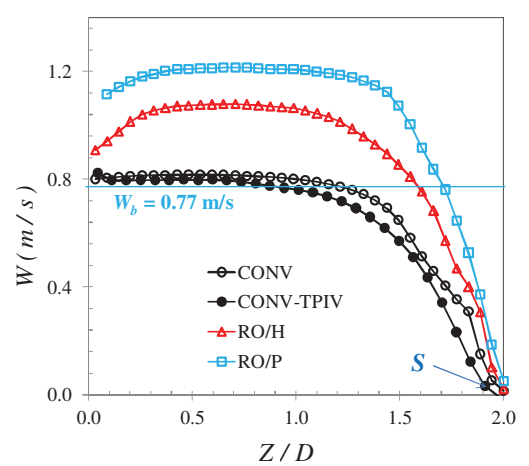

(b)

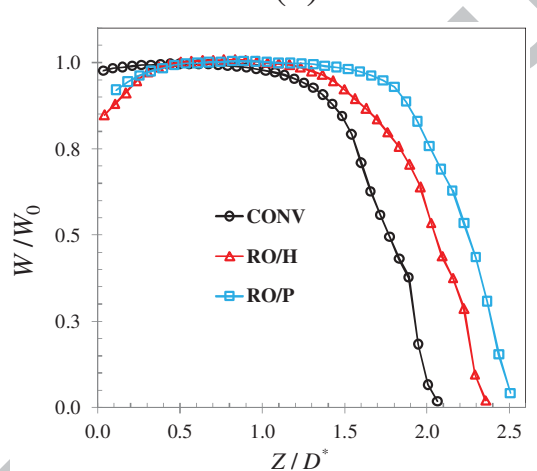

(c)

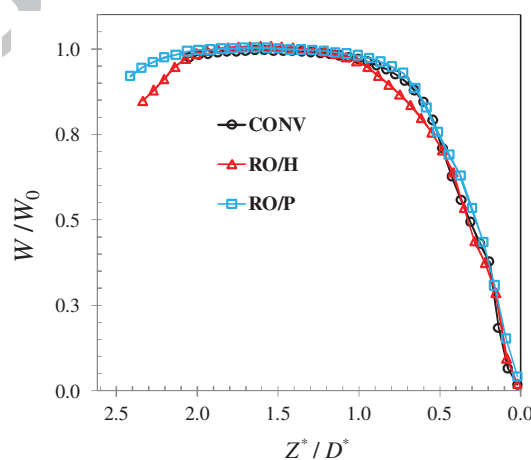

Figure 7: Streamwise mean velocity changes along the jet centerline; (a) with dimensional velocities; (b) using the second normalization, (b) using the second normalization and the origin of the jet axis positioned on the target wall 


\section{ACCEPTED MANUSCRIPT}

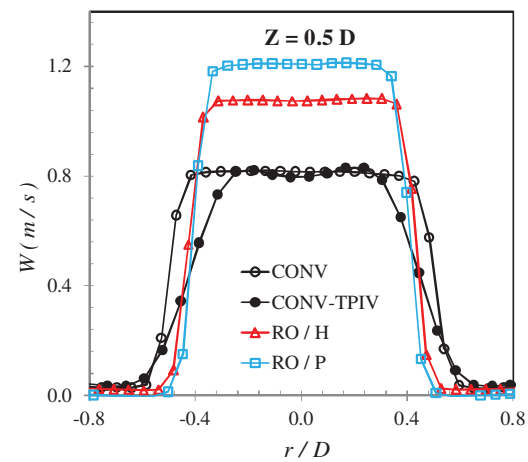

(a2)

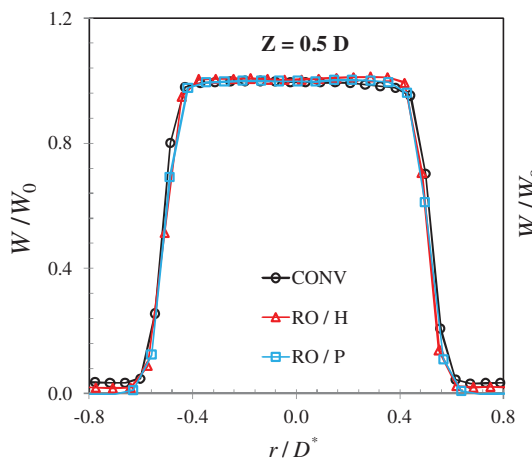

(a3)

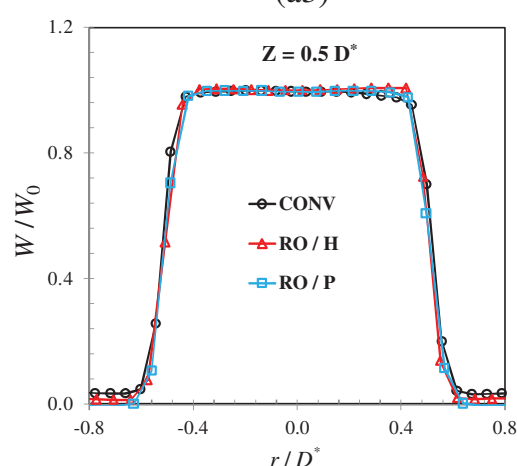

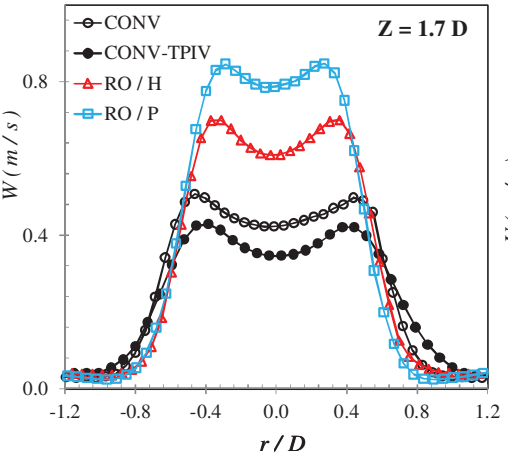

(b2)

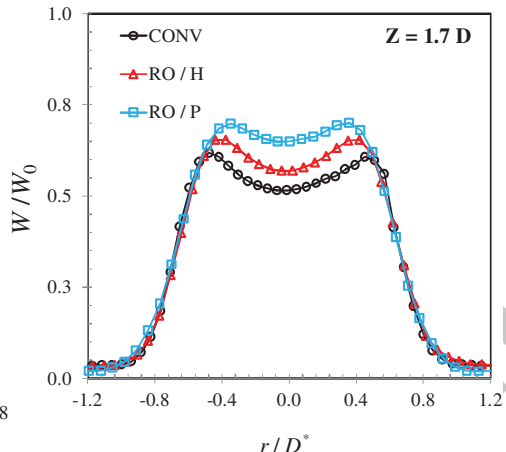

(b3)

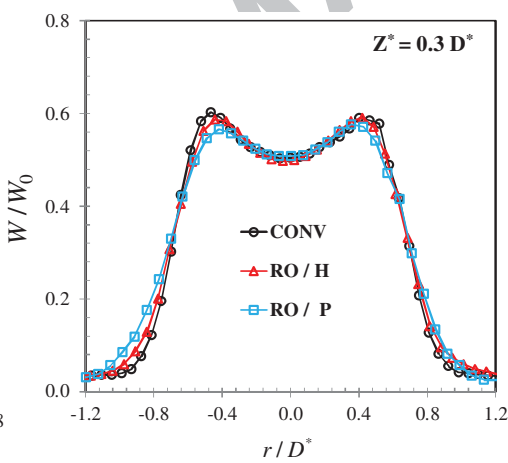

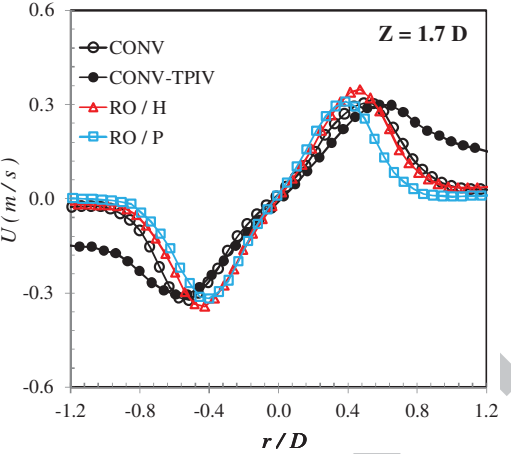

(c2)

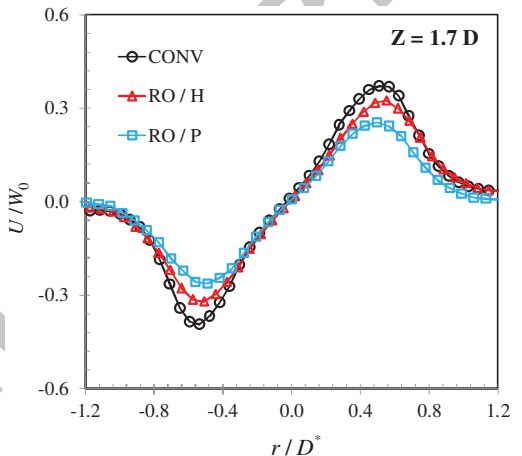

(c3)

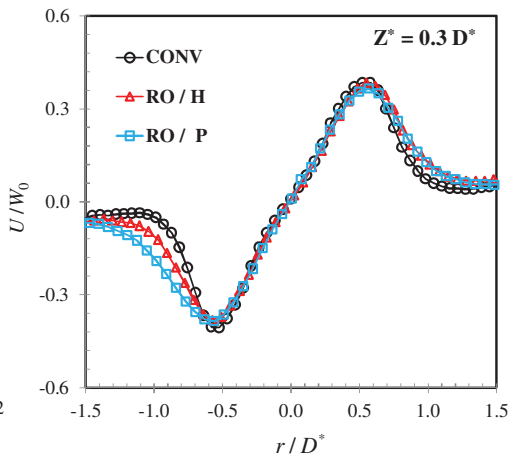

Figure 8: Streamwise velocity profiles ( $a, b)$ and transverse velocity profiles (c) at different locations: (1) with dimensional velocities; (2) using the second normalization at Z/D constant; (3) using the second normalization at $Z / D^{*}$ constant (a3) or at $Z^{*} / D^{*}$ constant (b3, c3)

(a)

(b) 

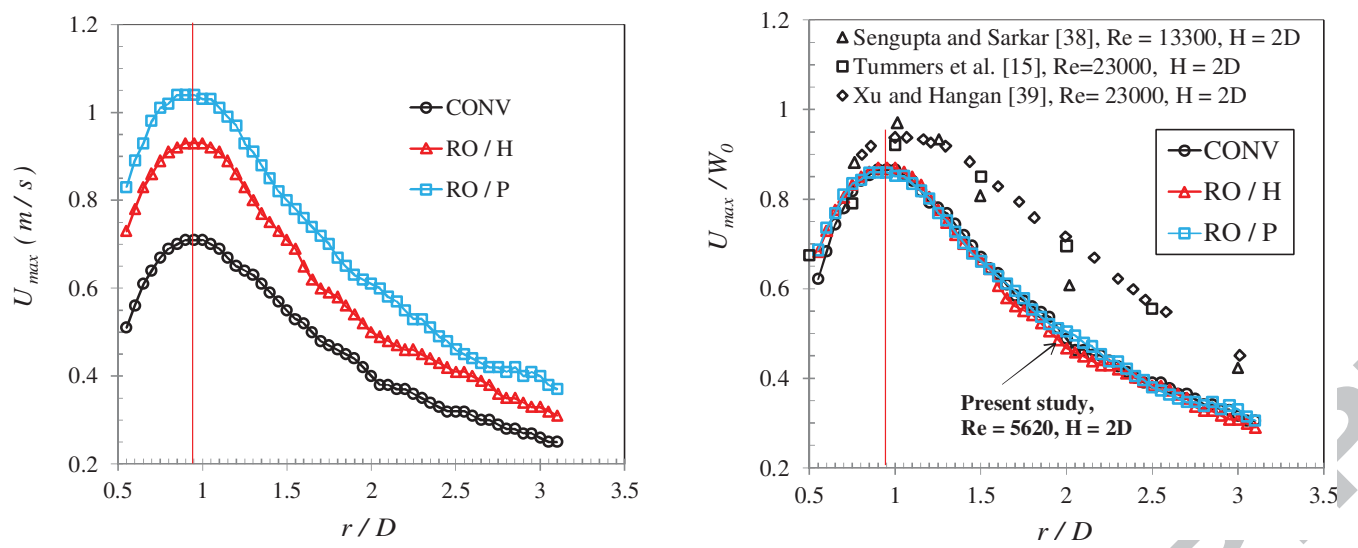

Figure 9: Radial distribution of maximum radial velocity above the wall. (a) dimensional values; (b) dimensionless values and comparison to the literature - the round nozzle in [15,39] is a pipe and in [38] a convergent connected to a short pipe

\subsection{Unsteady flow characteristics}

Prior to the analysis of the statistical properties of wall shear rate and mass transfer, the unsteady features of the flows were conducted based on electrodiffusion signals. These signals give information on the unsteady futures of impinging jet at the target which are expected to be related to the jet dynamics in its free region before impinging the wall. In the case of the reference convergent jet, we seek this possible link by the comparison of dominant frequencies in TPIV and ED signals.

Figure 10 provides the power spectra of electrodiffusion signals plotted against the non-dimensional frequency given by the Strouhal number $S t_{\theta}$. The Strouhal number is defined using the jet maximum velocity $W_{0}$ and the jet initial momentum thickness $\theta_{0}$ (Table 1) obtained for each jet using the mean streamwise velocity profile at $Z / D=0.25$. The power spectra were obtained by discrete Fourier transform of the electrodiffusion time-series signals. For clarity of representation, every spectrum is shifted two decades downwards with respect to the previous one.

For the radial location in the range $r=-0.4 D-1 D$, the spectra are displayed a hump centered on $S t_{\theta}=0.012$ for CONV nozzle jet, and on $S t_{\theta}=0.010$ for $\mathrm{RO} / \mathrm{H}$ and $\mathrm{RO} / \mathrm{P}$ nozzle jets. The corresponding frequencies are reported in Table 1.

In our previous work [21] that considered two of the nozzles of the present study at a lower Reynolds number $\operatorname{Re}_{b}=1360$, the normalized characteristic frequency of the wall shear stress fluctuations was 0.023 and 0.014 for the CONV nozzle and the RO/P nozzle, respectively. At the higher Reynolds number $\operatorname{Re}_{b}=$ 5620 considered here, the normalized characteristic frequency is 0.012 for the CONV nozzle and 0.010 for the RO/P nozzle. Although normalized characteristic frequencies values obtained in both studies falls in the range 0.009 to 0.023 given in the literature [40], they seem Reynolds number dependent and the dependency 
seems more important for the convergent nozzle compare to orifice nozzle. Husain and Hussain [41] observed for a given nozzle a decrease of the normalized characteristic frequency with increasing the shear layer Reynolds number $\operatorname{Re}_{\theta}$.

In our case, $R e_{\theta}=39$ in CONV and 42 in $\mathrm{RO} / \mathrm{P}$ for $R e_{b}=1360$ to be compared to $R e_{\theta}=148$ in $\mathrm{CONV}$ and 185 in $\mathrm{RO} / \mathrm{P}$ for $R e_{\mathrm{b}}=5620$. Hence, the increase of $R e_{\theta}$ is more important for $\mathrm{RO} / \mathrm{P}$ (4.4 times) compared to $\mathrm{RO} / \mathrm{H}$ (3.8 times) which could explain the difference in the changes of the corresponding $S t_{\theta}$.

The frequencies captured using the power spectra of the electrodiffusion signals are confirmed by the autocorrelation profiles displayed in Figure 11a at $r=1 D$. As it is well known, the autocorrelation and power spectrum have an inverse spreading relationship since both of these functions are Fourier transform pairs and the autocorrelation shifts out the strongest underlying event [42].

Figure $11 \mathrm{~b}$ compares for the CONV nozzle jet, the autocorrelation coefficient from ED to the one from TPIV. The TPIV signal is the time evolution of the transverse jet velocity $U$ at the position $Z / D=1.7$ and $r / D=0.6$. One can note that the two signals exhibit a same period. As it will be shown later on, the captured frequency corresponds to shedding phenomenon of the toroidal Kelvin-Helmholtz $(\mathrm{K}-\mathrm{H})$ structures.

The presence of the shedding frequency of K-H vortices in the ED signal is related to the imprint of these vortices on the target. Hence, the fundamental frequency in the ED signal for each jet (Figure 10) corresponds to the trace of the K-H vortices on the target.

In a free round jet [43], the $\mathrm{K}-\mathrm{H}$ vortices become three-dimensional after one or multiple pairing, and eventually break down at the end of the jet potential core which extends to $5-6 D$ [23]. In the present study, the target plate was placed at a distance $H=2 D$, where the structures were still well organized and no pairing took place. Beginning with the location $r=1.6 D$, the shedding frequency completely disappears for the three jets, meaning that the K-H vortices were far above the wall or they were already broken and destroyed. 


\section{ACCEPTED MANUSCRIPT}
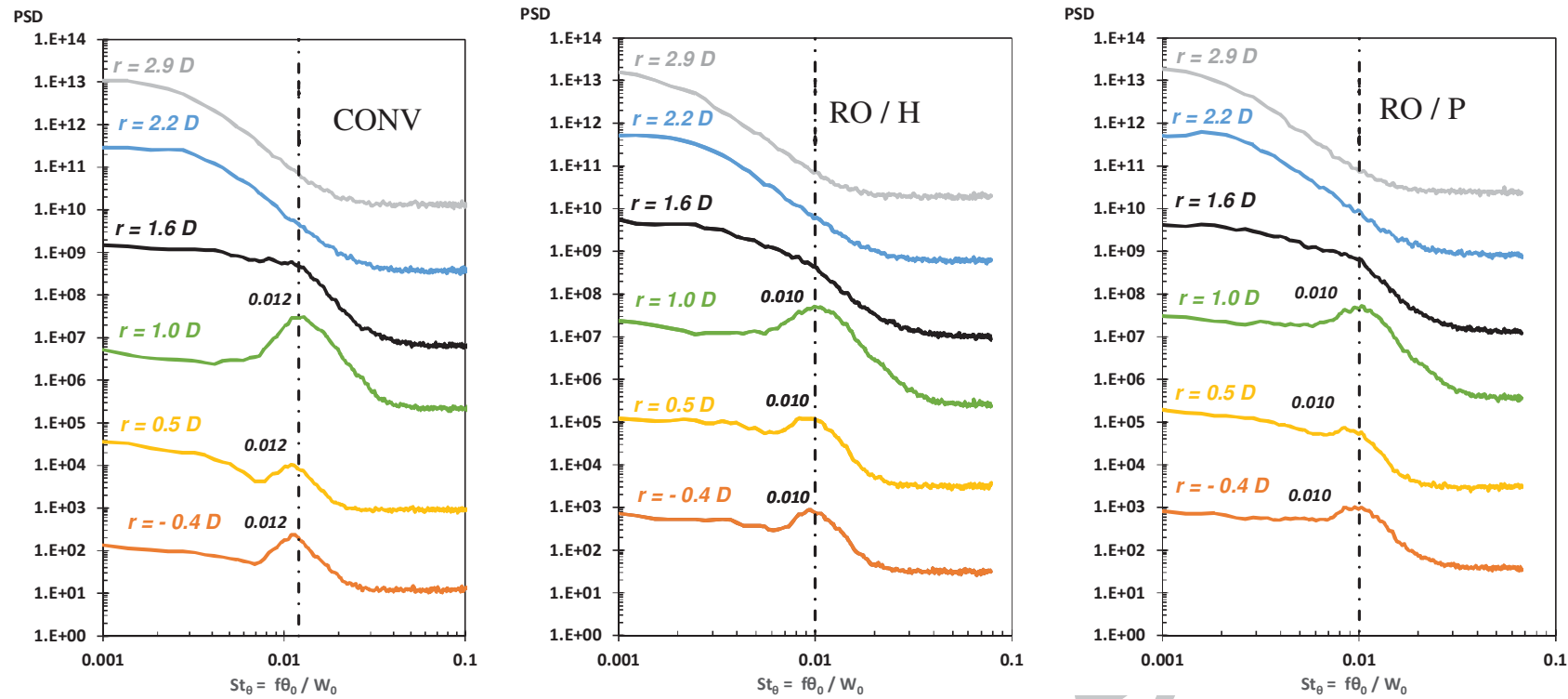

Figure 10: Power spectra of wall shear rate signals at different radial locations of the three jets- Every spectrum is shifted two decades downwards with respect to the previous. The vertical dashed line indicates the normalized characteristic frequency

(a)

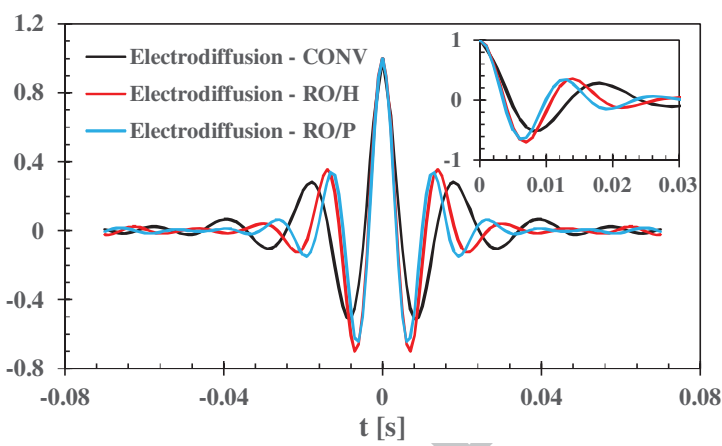

(b)

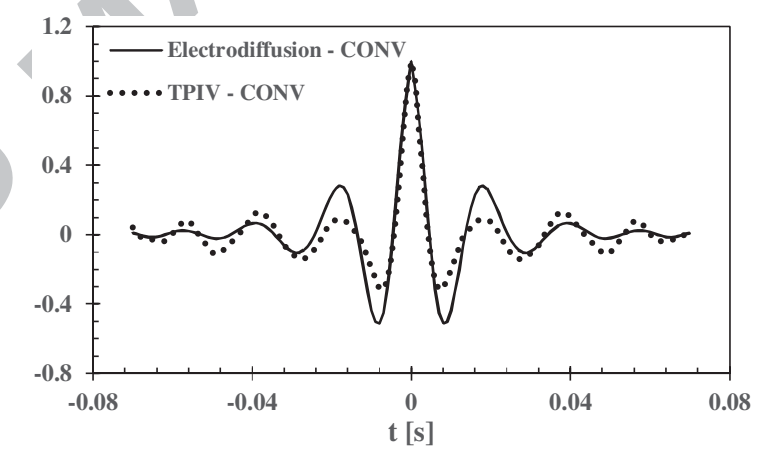

Figure 11: (a) Autocorrelation coefficient of the wall shear rate fluctuation at $r / D=1.0$ in the three jets; (b) Comparison to autocorrelation of the transverse velocity fluctuation at $r / D=0.6$ and $Z / D=1.7$ in the case of convergent jet

The three-dimensional flow organization of the CONV nozzle jet is given in Figure 12. This figure gives a temporal sequence of iso-surfaces of azimuthal vorticity $\omega_{\theta} D / W_{0}=2.5$ labeled "1" and "2". The time interval between the displayed snapshots is $\Delta t W_{0} / D=0.32$. The three dimensional behavior generated by the flow defection near the target is visualized by the iso-surface of axial vorticity $\omega_{Z} D / W_{0}= \pm 1.1$. 

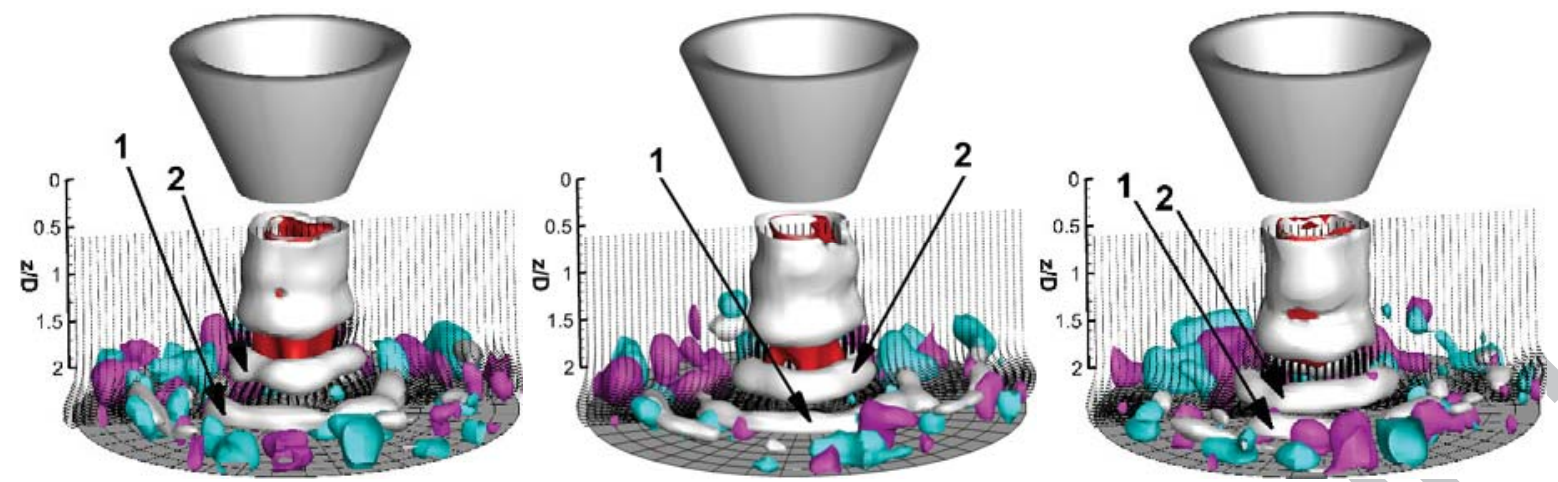

Figure 12: Time sequence instantaneous iso-surfaces of azimuthal vorticity $\omega_{\theta} D / W_{0}=2.5$ (White), axial vorticity $\omega_{Z} D / W_{0}=-1.1$ (cyan) and 1.1 (purple) and axial velocity $W=0.7[\mathrm{~m} / \mathrm{s}]$ (red), superimposed to the $Z-Y$ projection of velocity vector on the plane $X / D=0$ - The time interval between the displayed snapshots is $\Delta t W_{0} / D=0.32$

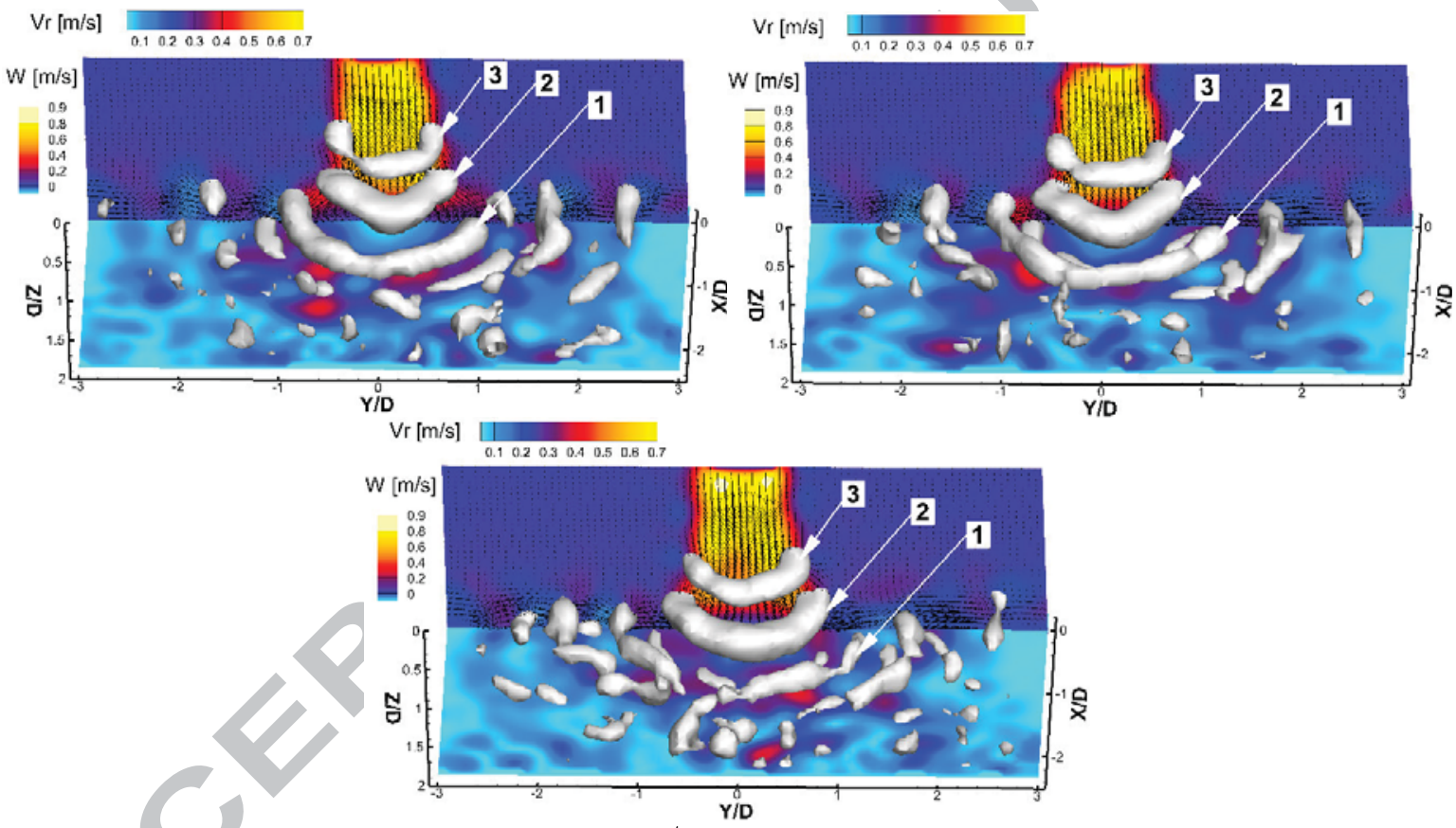

Figure 13: Time sequence of iso-surface of $\lambda_{2} D^{2} / W_{0}^{2}=-1.0$ (white), iso-contour of axial velocity $W$ on the vertical plane $X / D=0$, iso-contour of radial velocity $V_{r}$ at the vicinity of the plate $Z / D=1.9$, and $Z-Y$ projection of velocity vector. The time instants and order are the same as in Figure 12

Vortex structures are often identified from experimental velocity fields using the vorticity. However, the vorticity is not always convenient for this purpose as it cannot distinguish between pure shearing motion and swirling motion. A more pertinent method for vortex identification is the $\lambda_{2}$ - criterion based on the eigenvalues of the velocity gradient matrix. It uses the local pressure minimum criterion in the centre of a vortex [44]. This method was applied to the velocity fields of the Figure 12 and the results are given in 
Figure 13. This Figure also includes the contours of axial velocity $W$ along with velocity field on the vertical plane $X / D=0$ and radial velocity $V_{r}$ on the horizontal plane $Z / D=1.9$. The $\mathrm{K}-\mathrm{H}$ structure labeled " 3 " identified by the $\lambda_{2}$ - criterion in Figure 13 is not clearly identified by the vorticity contour in Figure 12 .

Figures 12 and 13 provide 3D view on the development of K-H instabilities in the initial shear layer, their roll-up and pinch-off and the advection of the resulting azimuthally coherent ring vortices. These vortices were shed between $Z / D=0.9$ and 1.4 (Figure 13) at a fundamental frequency of $53 \mathrm{~Hz}$. As mentioned above, this frequency is equal to that captured on the target using the electrodiffusion method. In the impinging region the ring vortices are still circular and less distorted until they strike the target. After the impact, as they are advected, they stretch in the radial direction, distort and break into small chips. As shown in Figure 14 providing a 2D view of the first snapshot from Figure 13, the resulting vortices are very close to the wall at $r / D=1.2$ and beginning with $r / D=1.5$, they draw far apart the wall. This observation is in agreement with the absence of periodicity in the wall shear rate signal at $r / D=1.6$ (Figure 10).

When the K-H vortices approached the wall, they stretched and their diameter increased (Figures 12 and 13). The instantaneous imprint of the $\mathrm{K}-\mathrm{H}$ vortices on the target, is visible in Figure 15 which gives the $\mathrm{X}-\mathrm{Y}$ presentation of the iso-lines $\lambda_{2} D^{2} / W_{0}^{2}=-1.0$ at $Z / D=1.8$ (i.e., at $1.6 \mathrm{~mm}$ from the target), along with isocolors of radial velocity $V_{r}$ and projection of velocity vectors on the plane X-Y. Popiel and Trass [45] using the smoke-wire flow visualization technique have shown that at nozzle-to-plate separation $H / D=1.2$, the toroidal vortices of the circular jet reached the plate at a radius $r=0.7 D-0.9 D$, which is consistent with the present results (Figure 15).

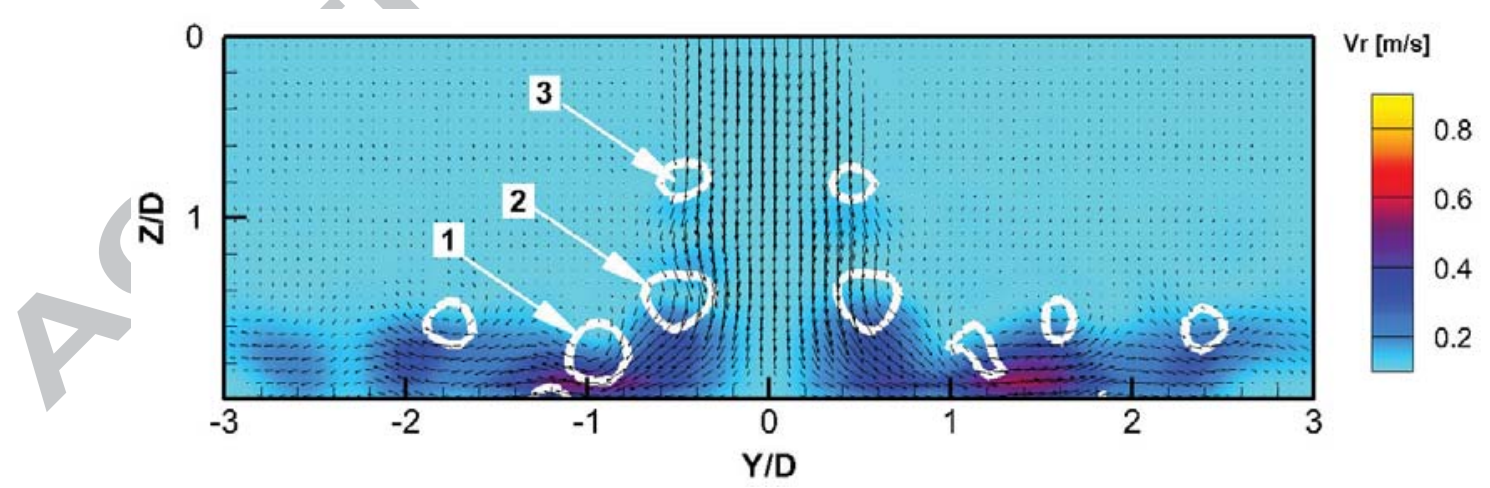

Figure 14: View in the vertical plane $X / D=0$ of the first instant from Figure 13-iso-lines of $\lambda_{2} D^{2} / W_{0}^{2}=-1.0$, iso-colors of the transverse velocity $V_{r}$ and velocity vector field in the plane 

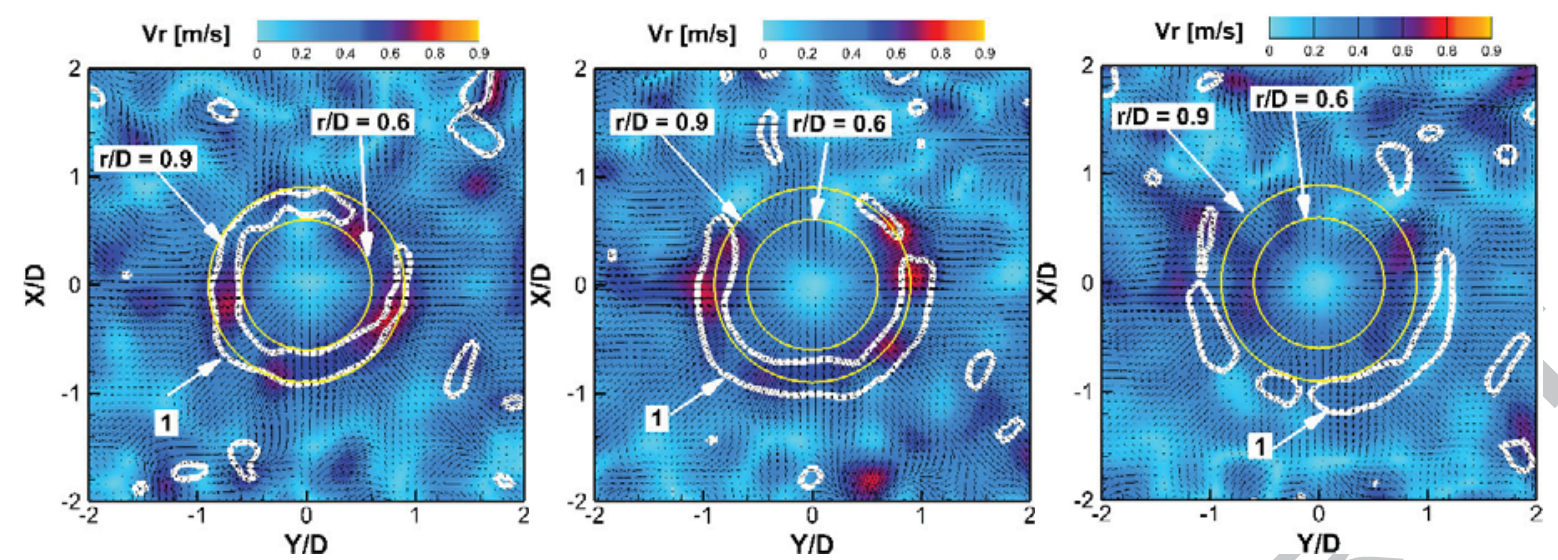

Figure 15: Time sequence of instantaneous iso-lines of $\lambda_{2} D^{2} / W_{0}^{2}=-1.0$ (white), iso-contours of radial velocity $V_{r}$ and $\mathrm{X}-\mathrm{Y}$ projection of velocity vector at $X / D=1.8$. The time interval between the displayed snapshots is $\Delta t W_{0} / D=0.42$

\subsection{Statistical properties of wall shear rate and mass transfer}

The radial distributions of the mean value of wall shear rate $\gamma$ (Eq. 7) and its RMS $\sqrt{\overline{\gamma^{\prime 2}}}$ are plotted in Figure 16 for the three considered nozzles. The maximum value of mean wall shear rate is equal to $9850 \mathrm{~s}^{-1}, 14670$ $\mathrm{s}^{-1}$ and $17380 \mathrm{~s}^{-1}$ for the CONV, RO/H and RO/P nozzle jet, respectively. Hence, the maximum of $\gamma$ is nozzle geometry dependent and is higher for the orifice jets than for the convergent jet at a Reynold number of 5620. This observation confirms the previous one made at a Reynold number of 1360 [21]. Contrary to our expectation, the hemispherical orifice nozzle $\mathrm{RO} / \mathrm{H}$ reduces somewhat $\gamma_{\max }$ level. That is probably because the curved surface already converge the flow at the exit and so reduces the vena contracta effect. Anyway, this finding is interesting for applications where wall-friction modulation is required. The curvature of the plate supporting the exhaust orifice may be modified to achieve a given level of local friction without changing the volumetric flow rate of the injected fluid.

Figure 17 a clearly shows the effect of nozzle geometry on the position of the maximum value $\gamma_{\max }$. It appears at $r=0.7 \mathrm{D}$ for the CONV nozzle jet, and at $r=0.58 \mathrm{D}$ and $0.55 \mathrm{D}$ in the $\mathrm{RO} / \mathrm{H}$ and $\mathrm{RO} / \mathrm{P}$ nozzle jet, respectively. These locations are close to the values $0.6 D-0.74 D$ reported in the literature [12-14] for the round impinging jets when $H<4 D$. Thus, the first peak of $\gamma$ does not coincide with the peak of $U_{\max }$ (Figure 9). The first peak of $\gamma$ is located in the growth region of $U_{\max }$.

Figure $17 \mathrm{a}$ also highlights in the stagnation region the changing of the $\gamma$-slope with the nozzle geometry. According to the method based on the assumption of uniform thickness of hydrodynamic and concentration boundary layer [20, 21], the stagnation mass transfer (Eq. 8) deduced from the slope of the radial wall shear rate distribution (Eq. 7) should be higher for orifice jets compared to the reference convergent jet. This is 
consistent with our previous observations made at very low Reynolds number $\operatorname{Re}_{b}=1360$ [21]. The stagnation mass transfer deduced from this indirect method will be compared further downstream to their direct measurements using the extend ED method proposed in the present study.

$\gamma=\partial u /\left.\partial z\right|_{z=0}=A \times r$

$S h=\frac{k D}{D_{C}}=\frac{1}{3^{1 / 3} \Gamma(4 / 3)}\left(\frac{A}{D_{C}}\right)^{1 / 3} D$

The wall shear rate falls sharply beyond its maximum (Figure 17 a) and then the decrease rate slows down in the region $r=1.1 D-1.8 D$; the slowing down is more pronounced in orifice jets. As shown in Figures 12 and 13, this region is dominated by a complex interaction between vortices issued from the breaking of the primary K-H vortices and the target wall. Hence, the difference between the convergent jet and the orifice jets regarding wall shear rate distribution is logically related to differences in vortex dynamics in the vicinity of the target wall. The radial distributions of the RMS of $\gamma$ normalized by its maximum value (Figure 16) are reported in Figure $17 \mathrm{~b}$. Whereas the RMS distribution of the convergent jet exhibits one sharp peak located at $1.1 D$, the orifice nozzle jets show a plateau ranging approximately from $0.8 D$ to $1.7 D$. This difference seems to be linked to the slope changing of $\gamma$ distribution in the same region (Figure 17a). Another interesting observation is the shift between the peak location of mean wall shear rate (Figure 17a) and its fluctuations (Figure 17b). In the case of the convergent jet, our normalized data $\gamma / \gamma_{\max }$ and $\sqrt{\overline{\gamma^{\prime 2}}} / \sqrt{\overline{\gamma^{\prime 2}}}$ max fit very well to those of Alekseenko et al. [46] also obtained by electrodiffusion technique. The authors provided both normalized mean wall shear rate and its RMS for a similar case than ours, i.e., a convergent nozzle jet at a Reynolds number $R e=6700$ and a nozzle to plate distance $H / D=2$. This comparison reinforces our findings regarding the shape of distribution of $\gamma$ and its RMS. Quantitative levels were not compared since it was not possible to trace the information on dimensional values of Alekseenko et al. due to the lack of information on their $\gamma_{\max }$ and ${\sqrt{\gamma^{\prime 2}}}_{\text {max }}$ values.

To move forward on the analysis of mean wall shear rate and its RMS distributions, the second normalization is applied (Figure 17 a' and b') and comparison is made with the data of the literature [13, 20, 47]. All authors consider the nominal nozzle diameter $D$ as a characteristic length scale of the jet and did not give the corresponding value of the characteristic diameter $D^{*}$. Anyway, in these studies the nozzle is either a convergent [13] or a pipe [47] for which the vena contracta effect is relatively low compared to the orifice nozzle. 
In another work [13] of Alekseenko et al. at a higher Reynolds number $R e=41600$, both mean wall shear rate and its RMS for a convergent nozzle jet at a nozzle to plate distance $H / D=2$ were provided and the given information were sufficient to apply to them the normalization considered in Figure 17 a' et b'.
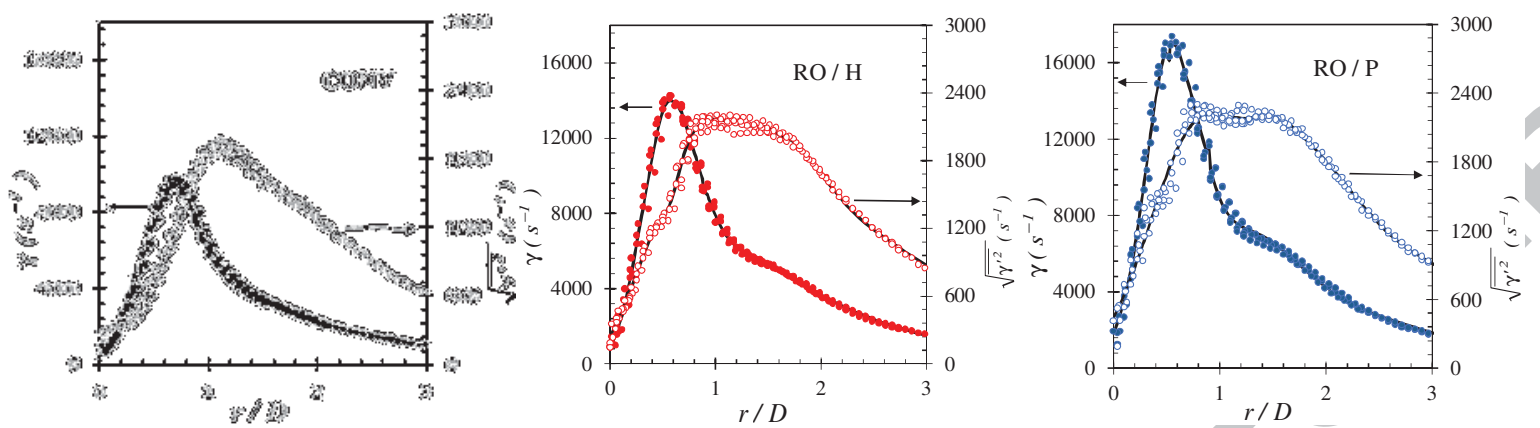

Figure 16: Wall shear rate and its RMS values as a function of normalized radial distance from the stagnation point

The normalized mean skin friction distributions (Figure $17 \mathrm{a}^{\prime}$ ) are similar for our three jets except at radial distance beyond $r / D^{*}=1.4$. In this region there is a slope change for orifice jets reflecting a birth of a second peak. The measurements of Alekseenko et al. [13] and Tummers et al. [47] that were performed at high Reynolds numbers are in good agreement with our results for $r / D^{*}<1.4$. Further downstream, the appearance of a high second peak supports the idea that the slope change in the skin friction distribution is $R e$-dependent. Indeed, as shown in Table 1 , in the present study at a constant $R e_{b}, R e$ is greater in orifice jets than in convergent jet due to the vena contracta effect and the corresponding jet acceleration.

Figure 17 b' compares in the second normalization form, the distributions of skin friction fluctuations in the three considered jets. Among the data sources used for previous comparisons made in Figure 17 a', only reference [13] provides the wall shear rate fluctuations. The data of reference [13] were obtained for a convergent nozzle at $R e=41600$ and $H / D=2$. Although the level and the position of normalized maximum fluctuation are different than ours for CONV nozzle jet explored at lower Reynolds number $(R e=5850)$, the distributions are similar in shape. Considering together, data from reference [46] at $R e=6700$ (Figure $17 \mathrm{~b}$ ) and data from reference [13] at $R e=41600$ (Figure 17 b'), differences in the distribution of normalized skin friction fluctuation in the convergent jet of Alekseenko et al. are surely due to Reynolds number level.

Considering our own results, the most noticeable feature evidenced by Figure $17 \mathrm{~b}$ ' is the particular flat distribution and the lower fluctuation values in orifice jets compared to those of a convergent jet. 
A similar trend is visible in velocity fluctuations near the target wall at $Z^{*} / D^{*}=0.3$ (Figure 18 a) and at $Z^{*} / D^{*}$ $=0.2$ (Figure $18 \mathrm{~b}$ ). At positions lower than $Z^{*} / D^{*}=0.2$, our PIV measurements are questionable due to the laser light scattering on the wall. Velocity fluctuations at $Z^{*} / D=0.04$ obtained by Vejrazka et al. [48] using hot wire anemometry in a circular convergent impinging jet $(R e=10000$ and $H / D=2)$ with or without external excitation are included in Figure $18 \mathrm{~b}$ for comparison. In the natural convergent impinging jet $\left(S t_{e}=0\right)$, the distribution of radial velocity fluctuation near the target wall present a peak around $Z / D=1$. This peak becomes sharper when the jet is excited at normalized frequency $S t_{e}=0.74$, whereas a plateau instead of local sharp peak appears at a normalized excitation frequency of $S t_{e}=2.14$. In this case, the plateau in velocity fluctuation is similar to the ones in the present study obtained for orifice jets. The authors observed that Kelvin-Helmholtz vortices produced in the jet at normalized excitation frequency $S t_{e}=0.74$ are large and regular, and while approaching the wall, they increase the near wall flow fluctuations. For normalized excitation frequency $S t_{e}=2.14$, Kelvin-Helmholtz vortices become small and disorganized that attenuate the near-wall flow fluctuations. In this case, the contribution of coherent vortices at the wall are similar to that of stochastic small scale fluctuating motion and lead to a plateau in velocity turbulent distribution.

Similar conclusions were drawn by Alekseenko et al.[46] concerning the effect of impinging jet excitation on transfer phenomena at the target wall. The authors observed that jet excitation at the most probable frequency (natural frequency) of the corresponding natural jet, enhances the coherence of the large vortices and increases the skin friction fluctuation with a maximum located around radial position $r=1.1 D$. By progressively suppressing the large scale vortices in the previous excited jet, using different concentrations of air bubbles introduced in the jet, they observed a strong local decrease of the skin friction fluctuation and the appearance of a plateau, instead of a local sharp peak in its radial distribution ranging from $1 D$ to $2.5 D$. On the other hand, when the jet without air bubbles is excited at frequencies exceeding the natural jet frequency, a similar plateau in the same region is also observed.

Based on the previous observations made in the literature about the link between coherent structures organization in the jet and the resulting radial distributions of wall shear stress [13] and the near-wall velocity fluctuations [48], it could be advanced that in orifice nozzle jets, coherent vortices are smaller and less regular than in the convergent jet, leading to their rapid breakdown on the target and to a plateau-type distribution and lower values of wall shear stress fluctuations (Figure $17 \mathrm{~b}$ and b'). 
In our previous work [21] on convergent and orifice impinging jets at the very low Reynolds number of 1360, such a difference in coherent structure organization in the two types of jets has been observed without making the link with near-wall fluctuations.

(a)

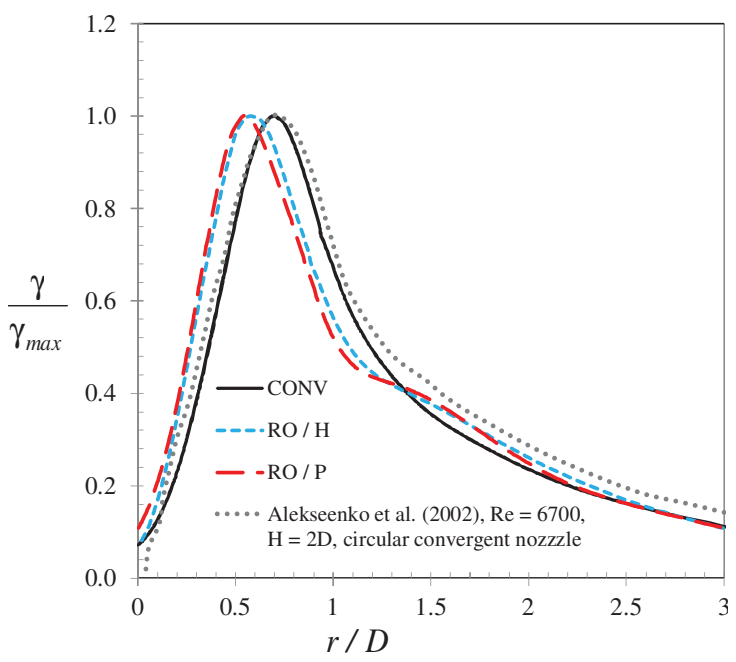

(a')

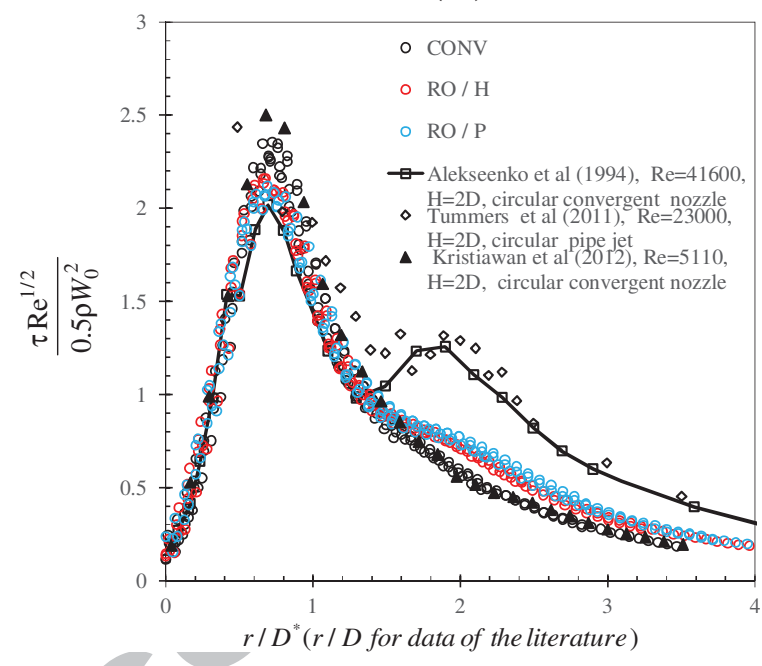

(b)

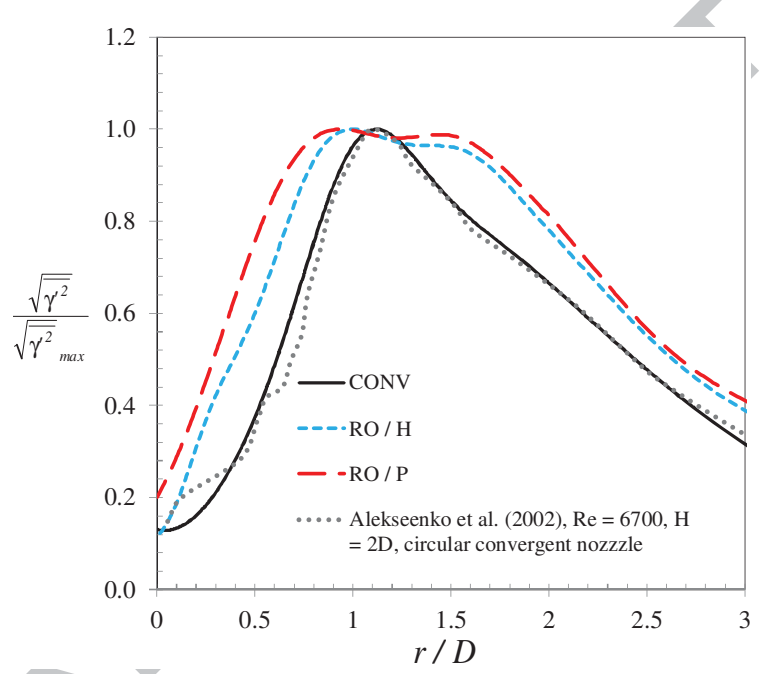

(b')

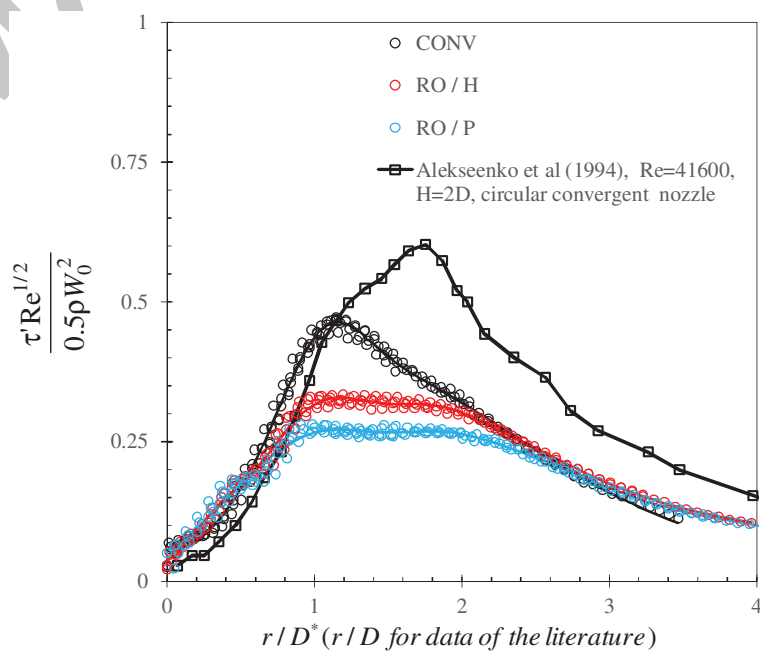

Figure 17: Radial distributions of normalized mean wall-shear rate (a, a') and of the corresponding mean square root (b, b') -comparison with available literature data 
(a)

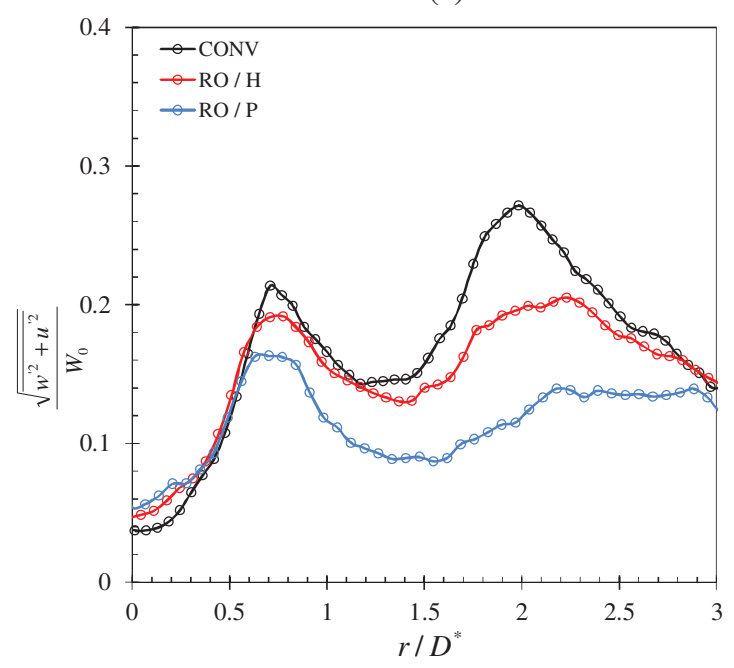

(b)

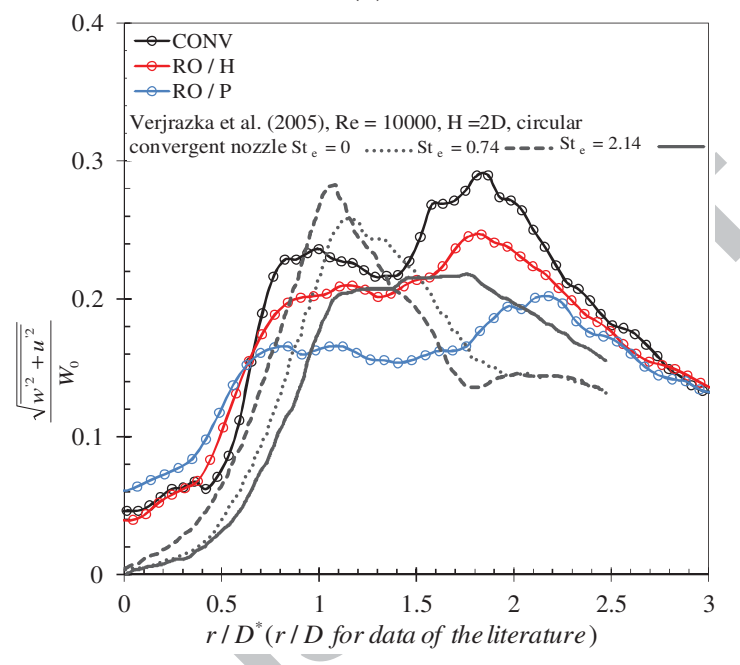

Figure 18: Radial distributions of normalized velocity fluctuation: (a) at $Z^{*} / D^{*}=0.3$; (b) at $Z^{*} / D^{*}=0.2-$ comparison with the data of Vejrazka et al.[48]

Figure 19 shows the radial distribution of the local Sherwood number $(S h)$ for the three considered jets. Similarly to what we observed for the wall shear rate $\gamma$, the maximum value of the local $S h$ is nozzle geometry dependent and is equal to 1042,1241 and 1392 for the CONV, RO/H and RO/P nozzle jet, respectively. The maximum Sherwood number $S h$ is then $19 \%$ and $34 \%$ higher for RO/H and RO/P nozzles compare to the CONV nozzle. Given that the maximum value of Sh corresponds to the maximum mass transfer, the present results demonstrate that the use of an orifice nozzle not only improves wall shear rate, but also increases the level of maximum mass transfer, which let assume that the latter is correlated to the former. The maximum of $S h$ occurs near the nozzle edge, approximately at $r=0.55 D, 0.45 D$ and $0.35 D$ for the $\mathrm{CONV}, \mathrm{RO} / \mathrm{H}$ and $\mathrm{RO} / \mathrm{P}$ nozzle jet, respectively. Hence, the first peak of $S h$ is shifted from the stagnation point, as was also observed by Kataoka et al. [12] in the core jet region of their convergent impinging jet. The authors explained that the mass transfer is very sensitive to the velocity turbulence which is still low on the jet axis in the potential core region and increases near the nozzle edge. In Vallis et al investigation [16], the peak of $S h$ for nozzle-to-plate distance in the range $5 D$ to $20 D$ appears on the stagnation point. It is expected here that the core jet region is already consumed at $5 D$, a position from which the turbulence rises on the jet axis. In numerous studies of heat transfer, as in Lee and Lee [49] and Colucci and Viskanta [50] investigations, the same behavior was observed for the Nusselt number $(\mathrm{Nu})$ distribution: the maximum of $\mathrm{Nu}$ in the core jet region is shifted from the stagnation point, whereas in the jet transition region and downstream the maximum of $N u$ appears at the stagnation point. 
Figure 19 highlights for the three jets what we might call "the birth of Sh secondary peak". The secondary peak in $S h$ distribution is more pronounced in orifice jets. It appears approximately at $r=2 D$ for the CONV nozzle jet, and at $r=1.7 D$ for the orifice jets.
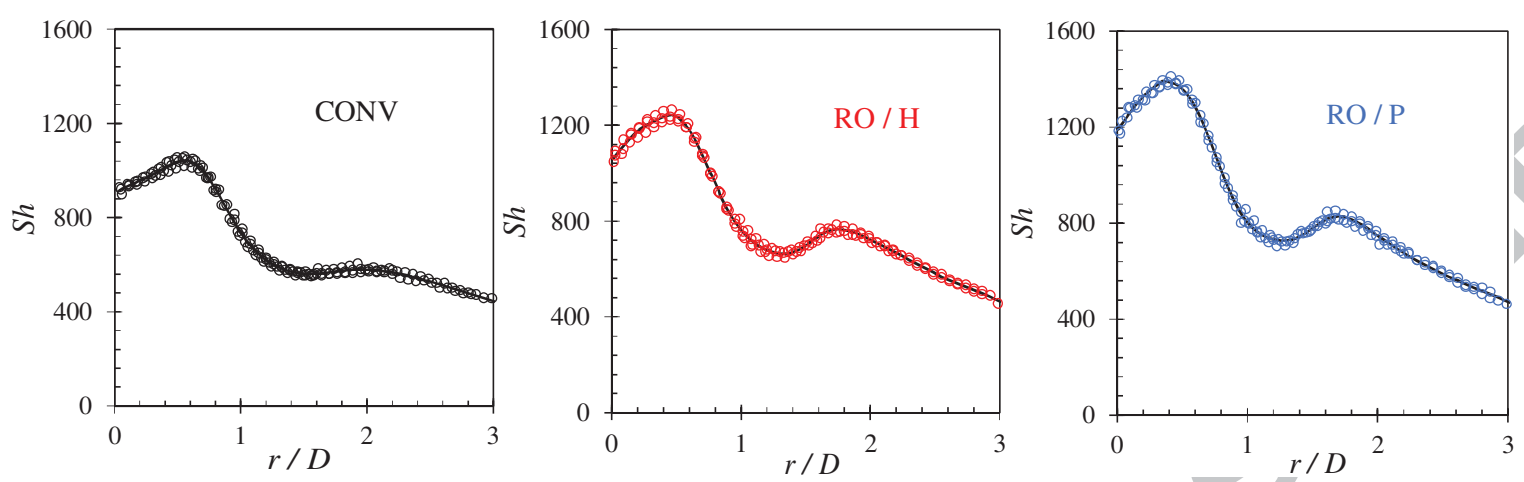

Figure 19: Local Sherwood number as a function of normalized radial distance from the stagnation point

As evidenced in Figure 20, the first peak in $S h$ distributions appears in the region where the K-H vortices strike the target (see also Figure 15) and its secondary peak emerges at the position where appear counterrotating vortices on instantaneous vorticity field; the secondary vortices are designated by an arrow in Figure 20 a. The secondary counter-rotating vortices are present in both convergent jet (Figure 20, left) and orifice jet (Figure 20, right). For brevity, only the orifice jet from RO/H nozzle is presented, because the RO/P case is similar.

If we advance the similarity between the secondary peak of $S h$ and the secondary peak of $N u$, the observation made above is consistent with that of Hadziabdic and Hanjalic [10]. From their LES simulation of a round impinging jet, the authors observed that the secondary peak in $\mathrm{Nu}$, (pertinent only for small $H / D$ and high Reynolds numbers) is caused by the reattachment of the recirculation bubble and by the associated turbulence production, as well as the subsequent strong advection. The conclusions of Hadziabdic and Hanjalic [10] are consistent with those of Carlomagno and Andrea [11] and Dairay et al.[51], who give in their recent works a comprehensive description of secondary vortex dynamics in impinging jets. 


\section{ACCEPTED MANUSCRIPT}

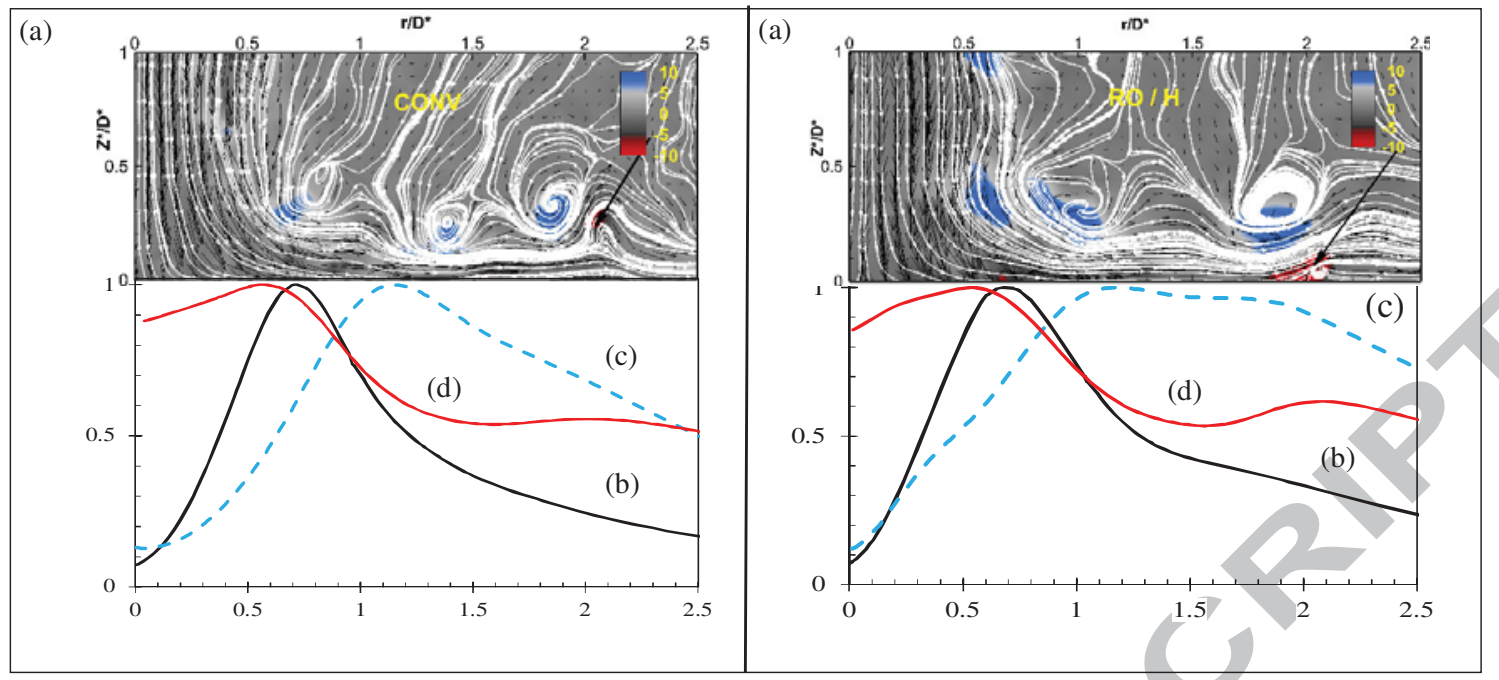

Figure 20: CONV nozzle jet (left) and RO/H nozzle jet (right): (a) Instantaneous vorticity field $\omega_{Y}=\left(\frac{\partial U}{\partial Z}-\frac{\partial W}{\partial X}\right) \frac{D}{W_{0}}-$ arrows indicate secondary vortices on the target; (b) $\gamma / \gamma_{\max }$; (c) $\sqrt{\overline{\gamma^{\prime 2}}} / \sqrt{\overline{\gamma^{\prime 2}}}{ }_{\max }$ (d) $S h / S h_{\max }$

Similarly to what was done previously for the skin friction (Figure 17), the Sherwood number is normalized using the two groups of parameters and the results are compared to the available literature data (Figure 21). When the Sherwood number is normalized using the characteristic scales (Figure 21 a), the profiles of the three jets collapse onto a single curve indicating that the jet contraction scales $W_{0}$ and $D^{*}$ are sufficient to model the difference on mass transfer introduced by the nozzle shape. The comparison of mass transfer results of the present study with the available literature data [52] obtained for convergent and orifice nozzles (Figure $21 \mathrm{~b}$ ) is made using nominal scales, because the authors do not provide the characteristic scales of their jets. Although maximum values and their positions in the two studies are almost identical in each type of jet, the overall agreement is less satisfactory. The origin of this difference could be attributed to the bulk Reynolds number $\mathrm{Re}_{\mathrm{b}}$ nearly four times greater in [52] than in the present study. 
(a)

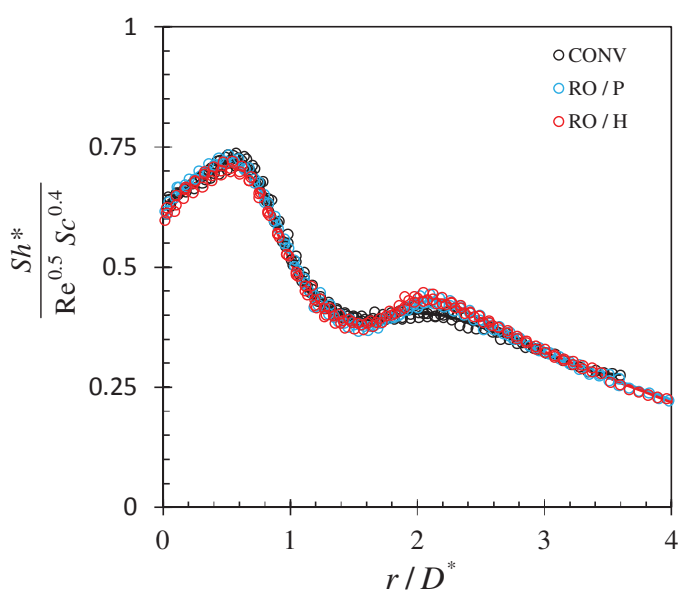

(b)

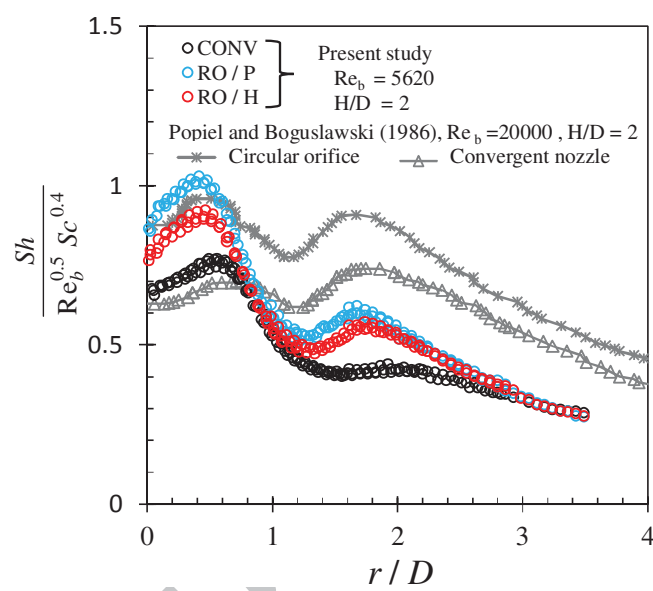

Figure 21: Normalized local Sherwood number distributions: (a) first normalization; (b) second normalization -comparison to the literature

One important quantity which has received considerable attention is the stagnation Sherwood number. In this study, the value of the stagnation Sherwood number is also nozzle geometry dependent (Figure 19). It is equal to 900, 1053 and 1154 for the CONV, RO/H and RO/P nozzle jet, respectively.

The stagnation Sherwood number can be also obtained from wall shear rate distribution as described in Kristiawan et al. [20] and Meslem et al. [21] and recalled above (see Eq. 7 and 8). As shown in Figure 22, the wall shear rates were fitted according to Eq. 7, with a linear dependence law in the stagnation region. The corresponding slope is equal to the hydrodynamic parameter A. The values of the stagnation Sherwood number calculated from the hydrodynamic parameter A using Eq. 8 is equal to 897, 1038 and 1140 for the $\mathrm{CONV}, \mathrm{RO} / \mathrm{H}$ and $\mathrm{RO} / \mathrm{P}$ nozzle jet, respectively. These values are very close to those obtained by the direct measurement procedure (Figure 19).

Chin and Tsang [17] proposed an empirical equation (Eq. 9) for the stagnation Sherwood number in the case of a turbulent straight pipe impinging jet with a Reynolds number ranging from 4000 to 16000 and a nozzleto-plate distance $H / D$ ranging from 0.2 to 6 .

$S h=1.12 \operatorname{Re}^{1 / 2} S c^{1 / 3} g(S c)(H / D)^{-0.057}$

Where $g(S c)$ is equal to 0.992 .

By extrapolating Eq. 9 to the parameters of the present study, we obtain a value of 898. Although the flow fields of a convergent jet and a pipe jet at a same Reynolds number could be different, the fact that our 
results are very close to the literature ones validates our measurements.

The normalized stagnation mass transfer coefficients, the ones obtained in the present study and in [21], are compared in Table 2 with the theoretical value of Shadlesky [53]. The deviation of our data relatively to the theoretical value falls in the range from 2 to $13 \%$, which is quite satisfactory.

Table 2: Normalized stagnation mass transfer coefficient $S h_{0}^{*} /\left(\operatorname{Re}^{0.5} S C^{0.5}\right)$, comparison with data of reference [21] and the theoretical value of reference [53]

\begin{tabular}{l|c|c|c} 
& $\mathrm{H} / \mathrm{D}=1$ & $\mathrm{H} / \mathrm{D}=2$ & $\mathrm{H} / \mathrm{D}=3$ \\
\hline CONV - Present study & - & 0.63 & - \\
\hline $\mathrm{RO} / \mathrm{P}$ - Present study & - & 0.60 & - \\
\hline $\mathrm{RO} / \mathrm{H}$ - Present study & - & 0.60 & - \\
\hline $\mathrm{CONV}$ - [21] & 0.61 & 0.63 & 0.66 \\
\hline RO / P - [21] & 0.63 & 0.61 & 0.60 \\
\hline Theoretical value [53] & 0.585 & 0.585 & 0.585
\end{tabular}
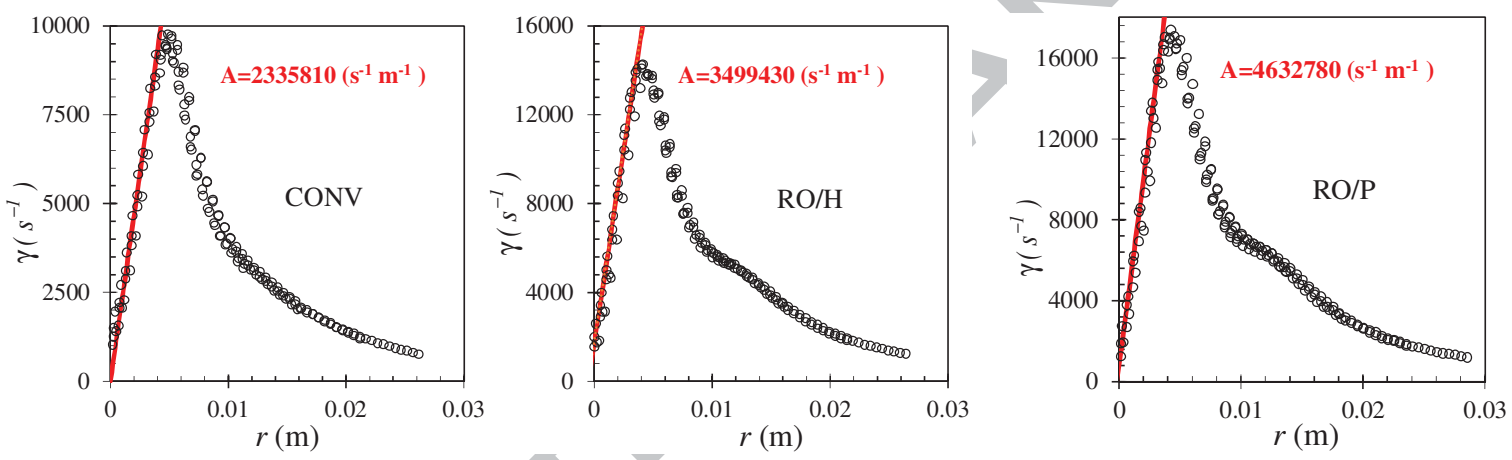

Figure 22: Determination of hydrodynamic parameter A from the wall shear rate profiles

Assuming the axial symmetry of the jets, the average Sherwood number $S h_{\text {avg }}$ can be obtained from the local Sherwood number as follows:

$S h_{\text {avg }}(r)=\frac{2}{r^{2}} \int_{0}^{r} \operatorname{Sh}(\rho) \times \rho d \rho$

For each jet, Figure 23a gives the best curve fit of the local Sherwood number and on Figure 23b, the radial distribution of the corresponding average value $S h_{\text {avg }}$ is plotted. The fitted curves were obtained using a cubic spline interpolation. The Figure 23 illustrates the mass transfer enhancement by the orifice jets not only locally (Figure 23a) but also globally in the disc area ranging from 0 to $3.5 D$ (Figure 23b). This trend was confirmed by direct measurement of the global Sherwood number obtained from the limiting diffusion current through the platinum disc of 3.2D (Figure 1b). The obtained values are 495, 618 and 650 for the 
$\mathrm{CONV}, \mathrm{RO} / \mathrm{H}$ and $\mathrm{RO} / \mathrm{P}$ nozzle jet, respectively. Their comparison to the corresponding integrated values 571, 648 and 680 obtained using Eq. 10 for $r=3.2 D$ (Figure 23b), reveals a difference of $15 \%$ for the convergent nozzle jet and 5\% for the orifice nozzle jets. Eq. 10 assumes an axial symmetry of the jet mean flow. This hypothesis seems to be less true for the convergent nozzle jet than for the orifice nozzle jets. Figure 24 showing the iso-contours of time-averaged axial velocity of the convergent jet in the $Y$ - $X$ plane at $Z / D=1.8$ (i.e. $1.6 \mathrm{~mm}$ above the target), confirms a defect in flow symmetry near the target that could explain the $15 \%$ difference between measured and calculated global Sherwood number.

(a)

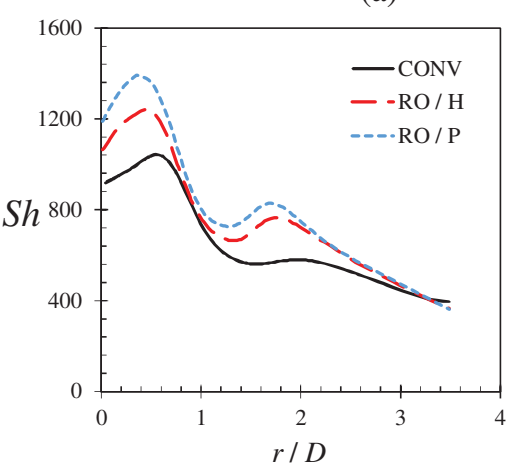

(b)

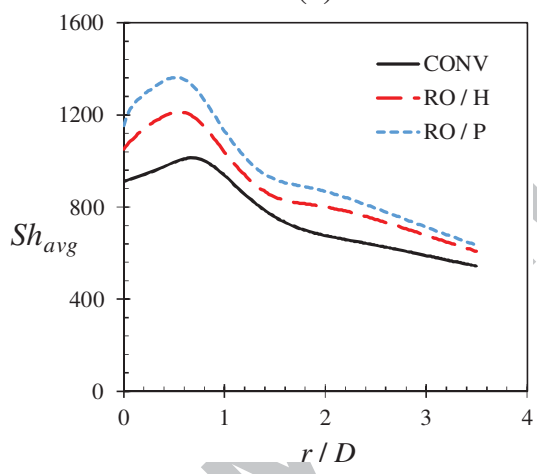

(c)

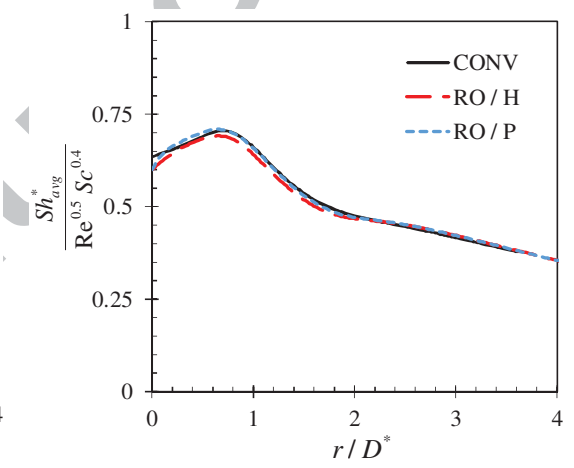

Figure 23: Curve fit of local Sherwood number distribution (a), calculated average Sherwood number (b) and calculated average Sherwood number in the second normalization form (c)

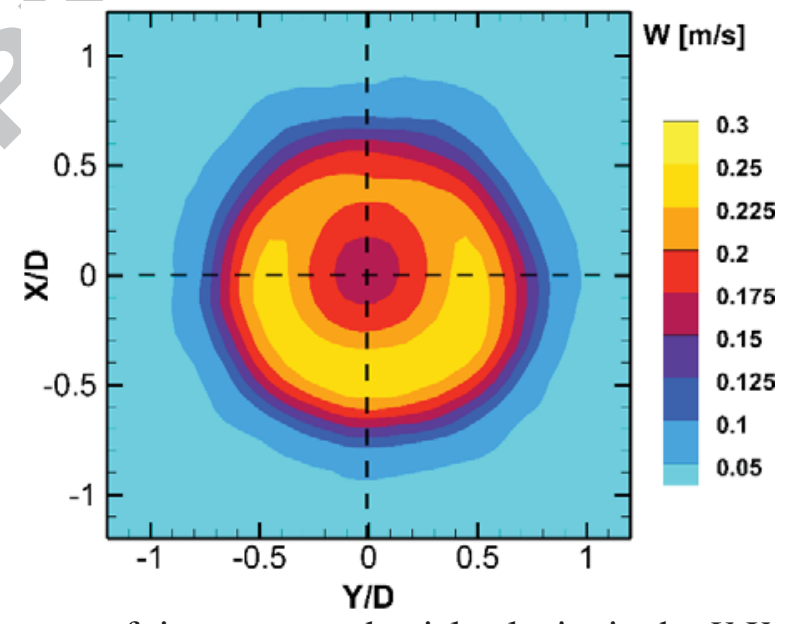

Figure 24: Iso-contours of time-averaged axial velocity in the $Y$ - $X$ plane at $Z / D=1.8$ for the convergent jet 


\section{Conclusion}

Three round impinging jets with exit nominal Reynolds number of 5620 and nozzle-to-wall distance of $2 D$, have been compared in the present study. A round orifice perforated either on a flat plate $(\mathrm{RO} / \mathrm{P})$ or on a hemispherical surface $(\mathrm{RO} / \mathrm{H})$, is compared to a reference convergent nozzle $(\mathrm{CONV})$. In each jet, Particle Image velocimetry (PIV) and Electrodiffusion technique (ED) were used to produce a data set on the flow field, the wall shear rate, and mass transfer, respectively. The study is conducted at constant exit area and volumetric flow rate for the three jets. This specific choice is related to the aimed Heating, Ventilation and Air Conditioning application, and specifically to the Personalized Ventilation aspect.

The instantaneous velocity fields indicated the formation of small secondary yortices above the impingement plate, under primary Kelvin-Helmholtz $(\mathrm{K}-\mathrm{H})$ in the region $r=1.5 D-2 D$, which is consistent with the literature. Consequently, beginning with $r=1.5 \mathrm{D}$, the structures issued from the breaking of the primary $\mathrm{K}-\mathrm{H}$ structures draw far apart the wall.

It was also shown that the wall shear rate fluctuation is related to the dynamics of the jet coherent structures. The shedding frequency of the K-H vortices revealed by time-resolved tomographic PIV, applied to the reference convergent jet (CONV), is present in the ED signals for $r<1.6 D$. At farther radial positions, the shedding frequency completely disappears from the ED signals whatever the jet considered, because the coherent structures are far above the wall or they are destroyed.

Jet mean field analysis reveals that the level of maximum velocity in the wall jet region is as high as the flow is accelerated at the jet exit. The jet acceleration was shown to be more intense in the orifice jets than in the convergent jet due to the vena contracta effect. However, the curved surface supporting the orifice stretches the flow at the exit, and reduces the vena contracta effect. This leads to a lower wall friction than in the case of the flat orifice. This finding is interesting for applications where wall-friction modulation control is required without changing the volumetric flow rate of the injected fluid.

It was shown that the use of an orifice nozzle not only improves wall shear rate, but also increases local and global mass transfer, which let assume that the latter is correlated to the former. At a constant volumetric flow rate and exit nozzle area, the global mass transfer on a target disc of $3.2 \mathrm{D}$ in diameter is $25 \%$ and $31 \%$ respectively higher for $\mathrm{RO} / \mathrm{H}$ and $\mathrm{RO} / \mathrm{P}$ nozzles compare to the reference $\mathrm{CONV}$ nozzle. As for local mass transfer, the local Sherwood number distributions indicate a gain of $19 \%$ and $34 \%$ with $\mathrm{RO} / \mathrm{H}$ and $\mathrm{RO} / \mathrm{P}$ nozzles compare to the CONV nozzle. Also, the distribution of the local Sherwood number exhibited a secondary peak in the wall region where secondary vortices appear. The level of this secondary peak is 
sensitive to the nozzle shape. The higher is the acceleration, the more intense is the level of the secondary peak.

The last main conclusion concerns the consequence of the choice of normalization parameters in data analysis. While large differences appear in nominal scale representations between the three jets, in both their statistical flow properties and their transfer processes, the use of characteristic scale representation clarifies these differences.

\section{ACKNOWLEDGMENT}

This work was supported by the Grants of the French National Agency of Research, project "FLUBAT", ANR-12-VBDU-0010

\section{REFERENCES}

1. R. Gardon, Heat Transfer Between a Flat Plate and Jets of Air Impinging on It. Int. Dev. Heat Transfer (ASME), 1962: p. 454-460.

2. R. Gardon and J.C. Akfirat, The role of turbulence in determining the heat-transfer characteristics of impinging jets. international Journal of Heat and Mass Transfer, 1965. 8: p. 1261-1272.

3. R. Gardon and J.C. Akfirat, Heat Transfer Characteristics of Impinging Two-Dimensional Air Jets. Journal of Heat Transfer, 1966. 88(1): p. 101-107.

4. C.O. Popiel and L. Boguslawski. Effect of Flow Structure on the heat or mass transfer on a Flat plate in Impinging Round jet. In 2nd UK National Conf. on Heat Transfer. 1988.

5. J. Lee and S.J. Lee, The effect of nozzle configuration on stagnation region heat transfer enhancement of axisymmetric jet impingement. International Journal of Heat and Mass Transfer, 2000. 43: p. 3497-3509.

6. K. Kataoka and T. Mizushina. Local enhancement of the rate of heat-transfer in an impinging round jet by free-stream turbulence. In Heat Transfer 1974; Proceedings of the Fifth International Conference, Tokyo, Volume 2. 1974. Tokyo.

7. D. Violato, A. Ianiro, G. Cardone, and F. Scarano, Three-dimensional vortex dynamics and convective heat transfer in circular and chevron impinging jets. International Journal of Heat and Fluid Flow, 2012. 37: p. $22-36$.

8. S. Roux, M. Fenot, G. Lalizel, L.E. Brizzi, and E. Dorignac, Experimental investigation of the flow and heat transfer of an impinging jet under acoustic excitation. International Journal of Heat and Mass Transfer, 2011. 54: p. 3277-3290.

9. D. Lytle and B.W. Webb, Air jet impingement heat transfer at low nozzle-plate spacings. International Journal of Heat and Mass Transfer, 1994. 37(12): p. 1687-1697.

10. M. Hadziabdic and K. Hanjalic, Vortical structures and heat transfer in a round impinging jet. Journal of Fluid Mechanics, 2008. 596: p. 221-260.

11. G.M. Carlomagno and A. Ianiro, Thermo-fluid-dynamics of submerged jets impinging at short nozzle-toplate distance: A review. Experimental thermal and fluid science, 2014. 58(0): p. 15-35.

12. K. Kataoka, Y. Kamiyama, S. Hashimoto, and T. Komai, Mass transfer between a plane surface and an impinging turbulent jet: the influence of surface-pressure fluctuations. Journal of Fluid Mechanics, 1982. 119: p. 91-105.

13. S.V. Alekseenko and D.M. Markovich, Electrodiffusion diagnostics of wall shear stresses in impinging jet. Journal of Applied Electrochemistry, 1994. 24: p. 626-631. 
14. D.J. Phares, G.T. Smedley, and R.C. Flagan, The wall shear stress produced by the normal impingement of a jet on a flat surface. Journal of Fluid Mechanics, 2000. 418: p. 351-375.

15. M.J. Tummers, J. Jacobse, and S.G.J. Voorbrood, Turbulent flow in the near field of a round impinging jet. international Journal of Heat and Mass Transfer, 2011. 54: p. 4939-4948.

16. E.A. Vallis, M.A. Patrick, and A.A. Wragg. Techniques of wall measurements in fluid mechanics. In Euromech.90. 1977. Nancy, France.

17. D.T. Chin and C.H. Tsang, Mass Transfer to an Impinging Jet Electrode. Journal of The Electrochemical Society, 1978. 125(9): p. 1461-1470.

18. M. El-Hassan, H. Hassan Assoum, V. Sobolik, J. Vétel, K. Abed-Meraim, A. Garon, and A. Sakout, Experimental investigation of the wall shear stress and the vortex dynamics in a circular impinging jet. Experiments in Fluids, 2012. 52(6): p. 1475-1489.

19. J.W. Hall and D. Ewing, On the dynamics of the large-scale structures in round impinging jets. Journal of Fluid Mechanics, 2006. 555: p. 439-458.

20. M. Kristiawan, A. Meslem, I. Nastase, and V. Sobolik, Wall shear rates and mass transfer in impinging jets: Comparison of circular convergent and cross-shaped orifice nozzles. International Journal of Heat and Mass Transfer, 2012. 55: p. 282-293.

21. A. Meslem, V. Sobolik, F. Bode, K. Sodjavi, Y. Zaouali, I. Nastase, and C. Croitoru, Flow dynamics and mass transfer in impinging circular jet at low Reynolds number. Comparison of convergent and orifice nozzles. International Journal of Heat and Mass Transfer, 2013. 67(0): p. 25-45.

22. Z. Bolashikov, A. Melikov, M. Spilak, I. Nastase, and A. Meslem, Improved inhaled air quality at reduced ventilation rate by control of airflow interaction at the breathing zone with lobed jets. HVAC\&R Research, 2013. 20(2): p. 238-250.

23. N. Rajaratnam, Turbulent jets. 1976, Amsterdam, Netherlands: Elsevier Scientific Publishing Company.

24. C.C. Landreth and R.J. Adrian, Impingement of a low Reynolds number turbulent circular jet onto a flat plate at normal incidence. Experiments in Fluids, 1990. 9: p. 74-84.

25. F. Scarano, L. Riethmuller M, and J. Adrian R, Advances in iterative multigrid PIV image processing. Experiments in Fluids, 2000. 29(3): p. S51-S60.

26. J. Westerweel, Theoretical analysis of the measurement precision in particle image velocimetry. Experiments in Fluids, 2000. 29(1): p. S003-S012.

27. R. Hain, C. Kähler, and D. Michaelis, Tomographic and time resolved PIV measurements on a finite cylinder mounted on a flat plate. Experiments in Fluids, 2008. 45(4): p. 715-724.

28. B. Wieneke, Volume self-calibration for 3D particle image velocimetry. Experiments in Fluids, 2008. 45(4): p. 549-556.

29. P.J. Schmid, D. Violato, and F. Scarano, Decomposition of time-resolved tomographic PIV. Experiments in Fluids, 2012. 52: p. 1567-1579.

30. G.E. Elsinga, F. Scarano, B. Wieneke, and B.W. van Oudheusden, Tomographic particle image velocimetry. Experiments in Fluids, 2006. 41(6): p. 933-947.

31. C. Atkinson, S. Coudert, J.-M. Foucaut, M. Stanislas, and J. Soria, The accuracy of tomographic particle image velocimetry for measurements of a turbulent boundary layer. Experiments in Fluids, 2011. 50(4): p. 1031-1056.

32. A.-J. Buchner, N. Buchmann, K. Kilany, C. Atkinson, and J. Soria, Stereoscopic and tomographic PIV of a pitching plate. Experiments in Fluids, 2012. 52(2): p. 299-314.

33. L.P. Reiss and T.J. Hanratty, Measurement of instantaneous rates of mass transfer to a small sink on a wall. AIChE Journal, 1962. 8(2): p. 245-247.

34. V. Sobolik, O. Wein, and J. Cermak, Simultaneous measurement of film thickness and wall shear stress in wavy flow of new-Newtonian liquids. Collection of Czechoslovak Chemical Communications, 1987. 52(4): p. 913-928.

35. V. Sobolik, J. Tihon, O. Wein, and K. Wichterle, Calibration of electrodiffusion friction probes using a voltage-step transient. Journal of Applied Electrochemistry, 1998. 28(3): p. 329-335.

36. W. Schabel and H. Martin, G10 Impinging jet Flow Heat Transfer, In VDI Heat Atlas. 2010, SpringerVerlag Heidelberg. p. 745-752. 
37. L.F.G. Geers, M.J. Tummers, T.J. Bueninck, and K. Hanjalić, Heat transfer correlation for hexagonal and in-line arrays of impinging jets. International Journal of Heat and Mass Transfer, 2008. 51(21-22): p. 53895399.

38. A. Sengupta and P.P. Sarkar, Experimental measurement and numerical simulation of an impinging jet with application to thunderstorm microburst winds. Journal of Wind Engineering and Industrial Aerodynamics, 2008. 96(3): p. 345-365.

39. Z. Xu and H. Hangan, Scale, boundary and inlet condition effects on impinging jets. Journal of Wind Engineering and Industrial Aerodynamics, 2008. 96(12): p. 2383-2402.

40. E.J. Gutmark and C.M. Ho, Preferred modes and the spreading rates of jets. Physics of Fluids, 1983. 26(10): p. 2932-2938.

41. Z.D. Husain and A.K.M.F. Hussain, Natural Instability of Free Shear Layers. AIAA Journal, 1983. 21(11): p. $1512-1517$.

42. $\quad$ K.B.M.Q. Zaman and A.K.M.F. Hussain, Vortex pairing in a circular jet under controlled excitation. Part 1. General response. Journal of Fluid Mechanics, 1980. 101(3).

43. C.G. Ball, H. Fellouah, and A. Pollard, The flow field in turbulent round free jets. Progress in Aerospace Sciences, 2012. 50: p. 1-26.

44. J. Jeong and F. Hussain, On the identification of a vortex. Journal of Fluid Mechanics, 1995. 285: p. 69-94.

45. C.O. Popiel and O. Trass, Visualization of a free and impinging round jet. Experimental Thermal and Fluid Science, 1991. 4: p. 253-264.

46. S.V. Alekseenko, D.M. Markovich, and V.I. Semenov, Turbulent Structure of a Gas-Liquid Impinging Jet. Fluid Dynamics, 2002. 37(5): p. 684-694.

47. M.J. Tummers, J. Jacobse, and S.G.J. Voorbrood, Turbulent flow in the near field of a round impinging jet. international Journal of Heat and Mass Transfer, 2011. 54(23-24): p. 4939-4948.

48. J. Vejrazka, J. Tihon, P. Marty, and V. Sobolík, Effect of an external excitation on the flow structure in a circular impinging jet. Physics of Fluids (1994-present), 2005. 17(10): p. -.

49. J. Lee and S.-J. Lee, The effect of nozzle aspect ratio on stagnation region heat transfer characteristics of elliptic impinging jet. international Journal of Heat and Mass Transfer, 2000. 43(4): p. 555-575.

50. W. Colucci D and R. Viskanta, Effect of nozzle geometry on local convective heat transfer to a confined impinging air jet. Experimental Thermal and Fluid Science, 1996. 13(1): p. 71-80.

51. T. Dairay, V. Fortuné, E. Lamballais, and L.-E. Brizzi, Journal of Fluid Mechanics, 2015. 764, : p. 362-394

52. C.O. Popiel and L. Boguslawski. Mass or heat transfer in impinging single, round jets emitted by a bellshaped nozzle and sharp-ended orifice. In International Heat Transfer Conference 8. 1986. San Francisco, USA.

53. P.S. Shadlesky, Stagnation point heat transfer for jet impingement to a plane surface. AIAA Journal, 1983. 21(8): p. 1214-1215. 


\section{Research Highlights:}

$>$ PIV and Electrodiffusion techniques were used to characterize a round impinging jet $>$ three nozzle geometries were considered with a same bulk Reynolds number > It is shown that wall shear rate and local or global mass transfer are nozzle geometry dependent $>$ The jet issued from an orifice perforated on a flat plate was found more efficient than a jet from an orifice perforated on a hemispherical surface or from a convergent nozzle. 